\title{
C++ TOOLS TO CONSTRUCT OUR USER-LEVEL LANGUAGE
}

\author{
FRÉDÉRIC HECHT ${ }^{1}$
}

\begin{abstract}
The aim of this paper is to present how to make a dedicaded computed language polymorphic and multi type, in $\mathrm{C}++$ to solve partial differential equations with the finite element method. The driving idea is to make the language as close as possible to the mathematical notation.
\end{abstract}

Mathematics Subject Classification. 68N15, 78M10, 80M10.

Received: January 10, 2001. Revised: May 23, 2002.

\section{INTRODUCTION}

A partial differential equation is a relation between a function of several variables and its (partial) derivatives. Many problems in physics, engineering, mathematics and even banking are model by one or several partial differential equations.

Many phenomena involve several different fields. Fluid-structure interactions, Lorentz forces in liquid aluminum and ocean-atmosphere problems are three such systems. These require approximations of different degrees, possibly on different meshes. Some algorithms such as Schwarz' domain decomposition method require also the interpolation of data on different meshes within one program. Thus the software freefem++ [6] can handle these difficulties: arbitrary finite element spaces on arbitrary unstructured and adaptive meshes (see $[2,9]$ for the finite elements method).

We present the kernel of the freefem++ user-level language. First, this language as to be able to use functions. In Section 2, we explain how to construct an algebra of functions, in Section 2.2 we generalize this construction to $\mathcal{C}^{\infty}$ functions, and in Section 3, we explain how to modelised elliptic PDE's in variational form, and how to compute integrals.

To construct our user-level language, we need a virtual machine to execute the instructions. Wirth's book [11] explains clearly the way. The way to build the virtual machine is based on the the following remark: the algebra of functions is a simple version of a virtual machine with just $+,-, *, /, \wedge$ operator, where the "run" action is the evaluation of the function. To construct a virtual machine, we just add to the algebra of functions, some operators and functions like:

, sequencing operator

$=$ affectation operator

while while loop construction

getvalue get the value of local variable

call call a virtual machine function

$\cdots$

Keywords and phrases. Finite element method, grammars, languages.

${ }^{1}$ Laboratoire Jacques-Louis Lions, Université Pierre et Marie Curie, Paris, France. e-mail: hecht@ann.jussieu.fr 
The virtual machine is an extended algebra of "function instructions", with lot of operators and functions. All the local variables of a procedure are store in a stack memory. We want a reentrant language so the memory stack must be a parameter of the "function instructions" and this stack must be allocated when we evaluated the operator "call" a function.

We describe how to change the algebra, to be able to handle many data types, with or without dynamic type checking. We explain how to use the STL to make symbol tables and how to handle easily the polymorphism in the language. We finish with the description of how add operators to the user language, and how to use simply yacc or bison processor.

Finaly, we give two example from the freefem++ language, one to solve the Navier-Stokes equation (Chap. 5 in [10]) by the Taylod-Hood approximation, and another for the Schwarz' domain decomposition method [8].

\section{An Algebra of functions}

In this section we show how to construct an algebra of function in $C_{++}$. We start, with functions from $\mathbb{R}$ to $\mathbb{R}$, a mathematical function from $\mathbb{R}$ to $\mathbb{R}$, is just an object with a name, $f$ for example, with the evaluation operator $f(x)$ for any $x \in \mathbb{R}$.

\subsection{Basic version}

First $^{1}$, we just make an algebra of functions with the classical mathematical operators,,+- * , / , ^. The model function is just a pure virtual class Cvirt with only the evaluation operator: $R$ operator() (R) const; .

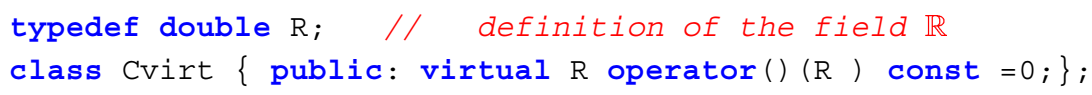

Now we use inheritence, to create the classical functions ( +++ function, constant function, combination of two functions).

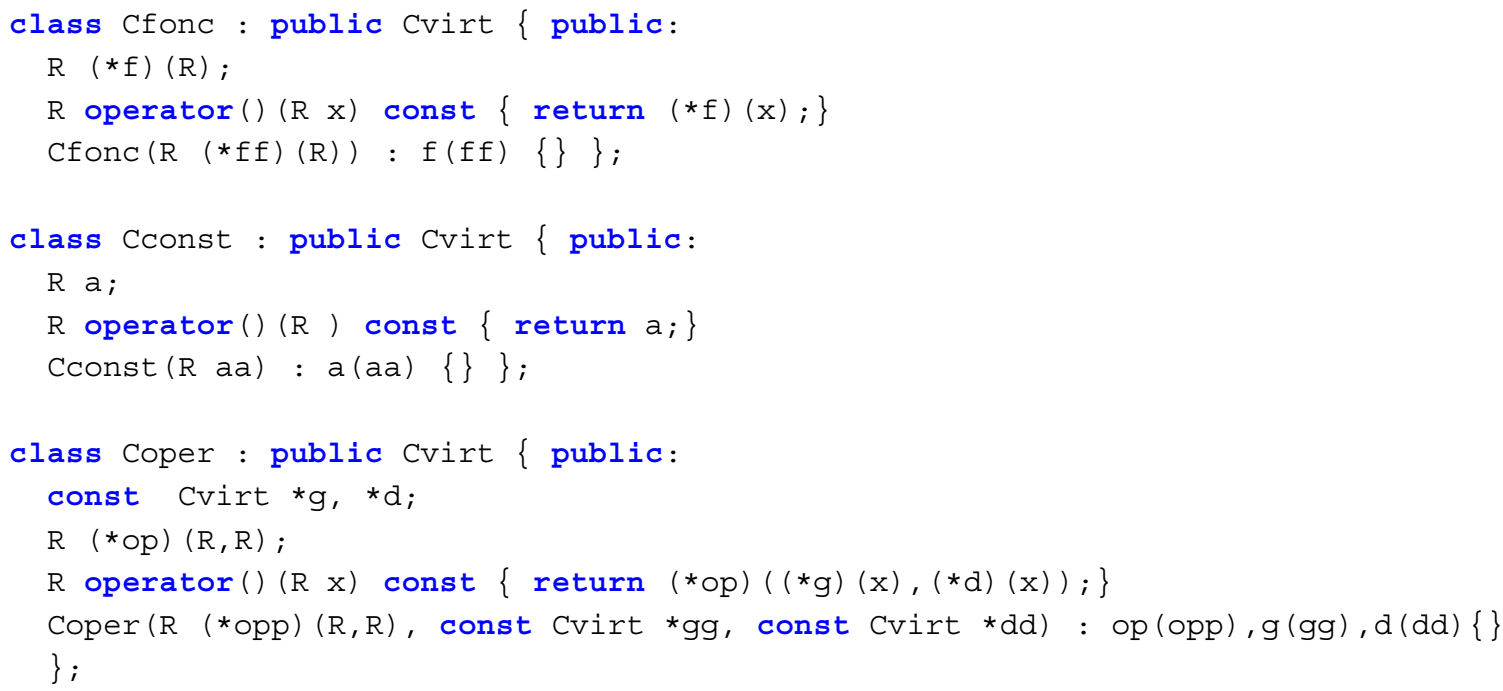

We define the five classical operators.

// the 5 binary operators

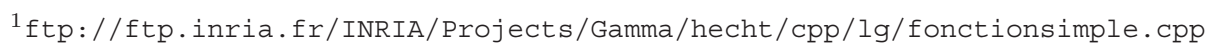




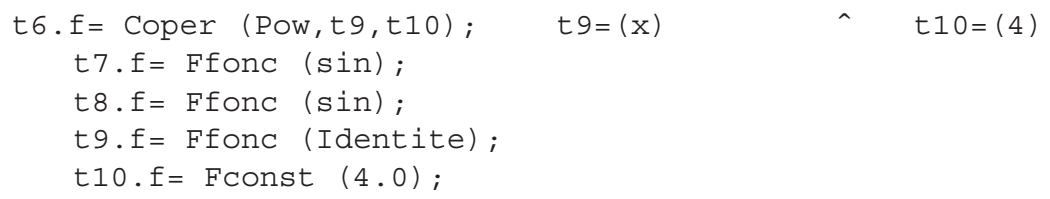

\subsection{The $C^{\infty}$ function}

Now in this version ${ }^{2}$, we want to differentiate the function recursively, this tool is also use in FreeFem+ software [1]. We focus on the problem of infinite recursively, if the function constructor build also the derivative, recursively all the derivative of the function will be effectively constructed. The n-derivative of function $x \rightarrow 1 / x$ is $(-1)^{n}(n !) x^{-n-1}$, when we construct the function $1 / x$, all the derivative will be also constructed, we get a "infinite program" (no bug at the compilation time, just a stack overflow at execution).

To solve this, we use a construction in two stages. First choose a variable (md, initialize to 0 ) to store the derivative and secondly use the virtual method virtual CVirt *de () \{return zero; construct the the derivative function. Now, the method CVirt $* \mathrm{~d}()$, can construct the derivative function once only when we need it.

So the class is:

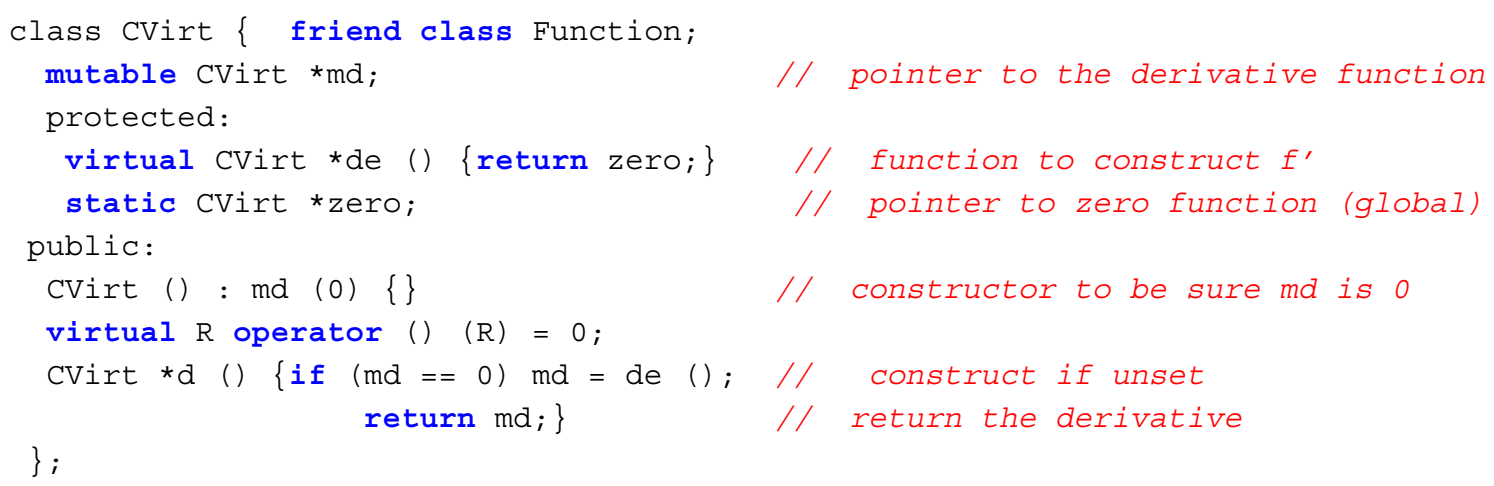

We also introduce the functions Function 2 from $\mathbb{R}^{2}$ to $\mathbb{R}$. and we use the composition operator to defined all the derivative of the binaries operators (example: operator $f+g=\operatorname{Add}(f, g)$ ).

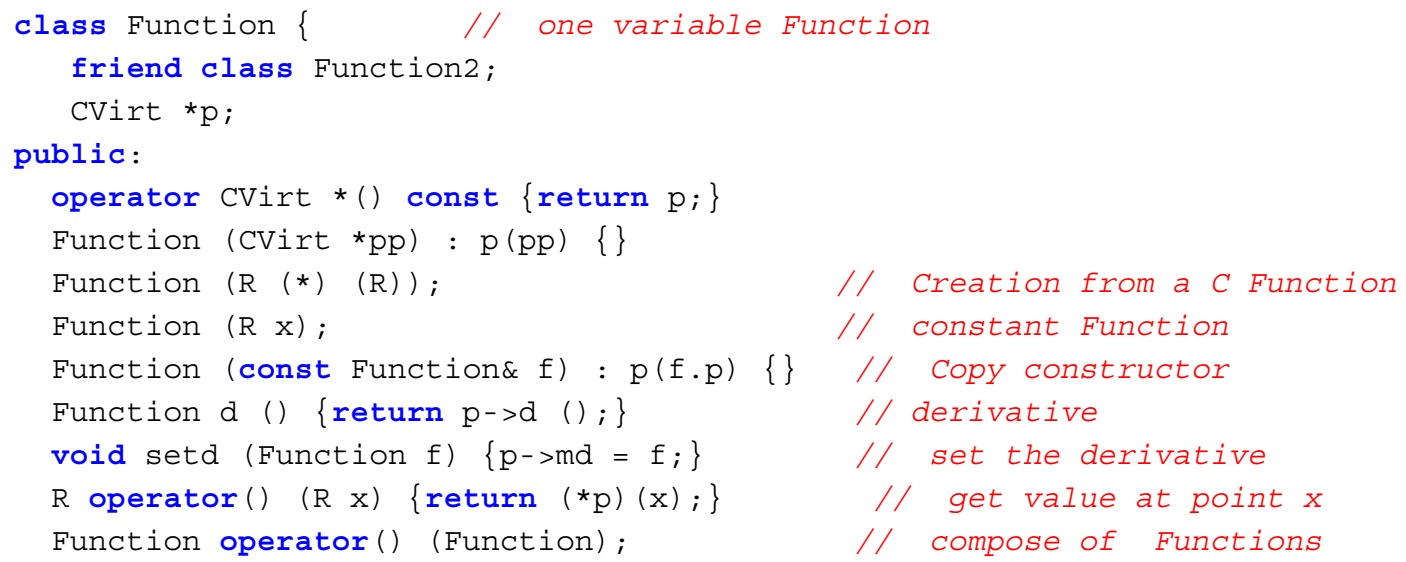

${ }^{2}$ ftp://ftp.inria.fr/INRIA/Projects/Gamma/hecht/cpp/lg/Function. [ch] pp 


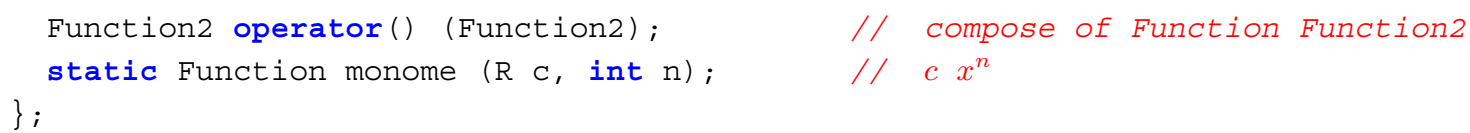

With the same technique, the virtual class for functions from $\mathbb{R}^{2}$ to $\mathbb{R}$ is:

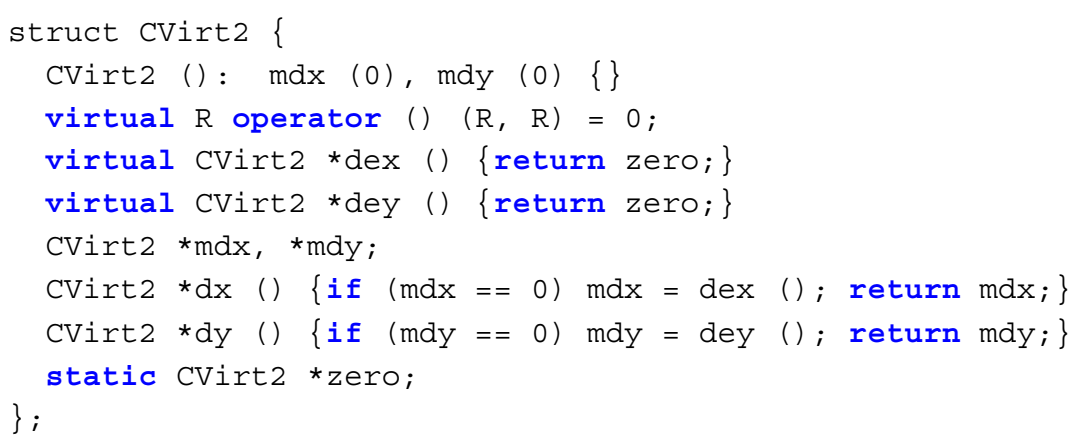

Now the class of functions from $\mathbb{R}^{2}$ to $\mathbb{R}$ is:

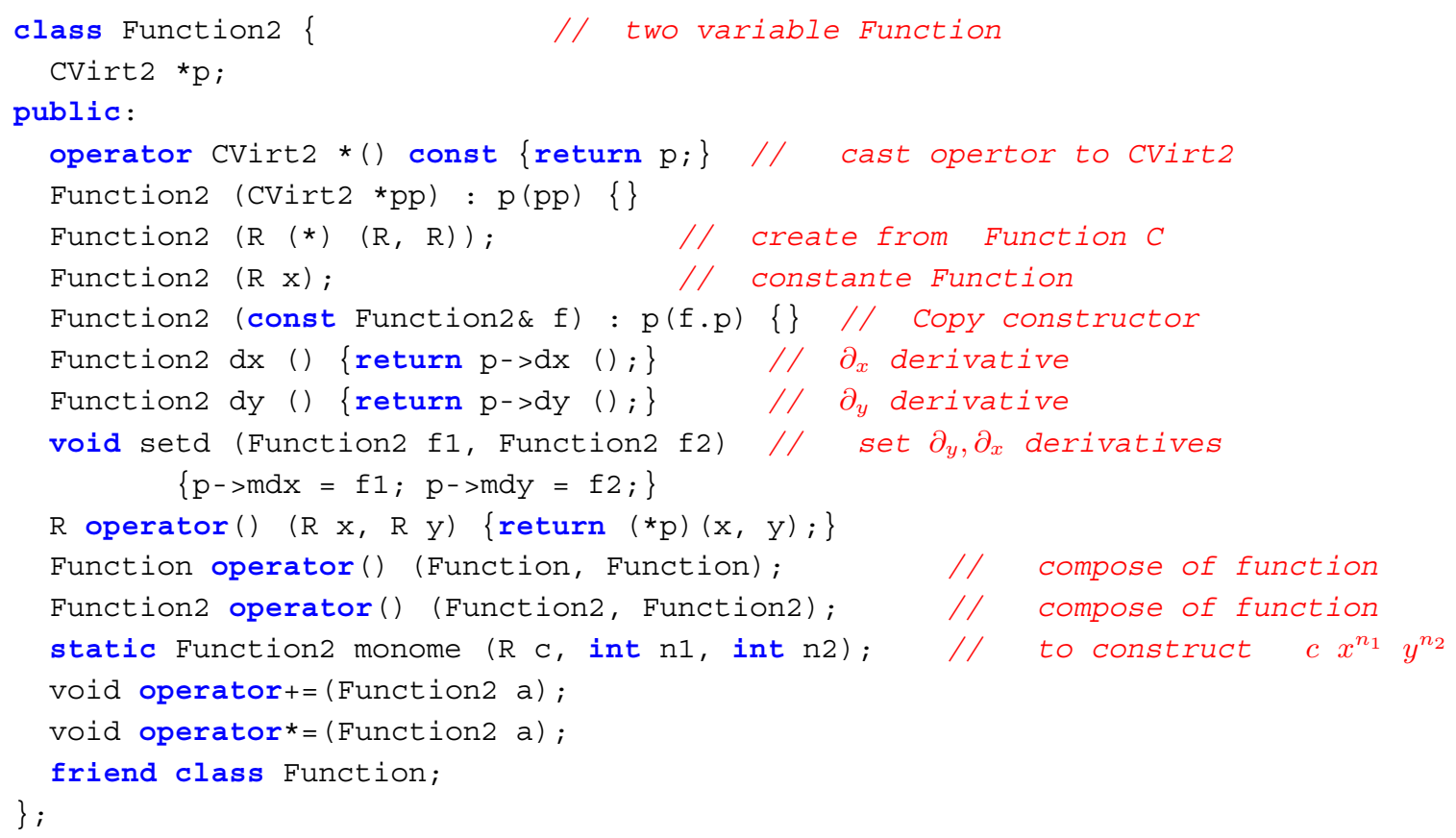

Some useful global functions:

extern Function Chs, Identity, Log;

extern Function2 Add, Sub, Mul, Div, Pow, CoordinateX, Coordinatey;

All the operators are:

inline Function operator+ (Function f, Function g) \{return Add(f, g); inline Function operator- (Function f, Function g) \{return Sub(f, g); 


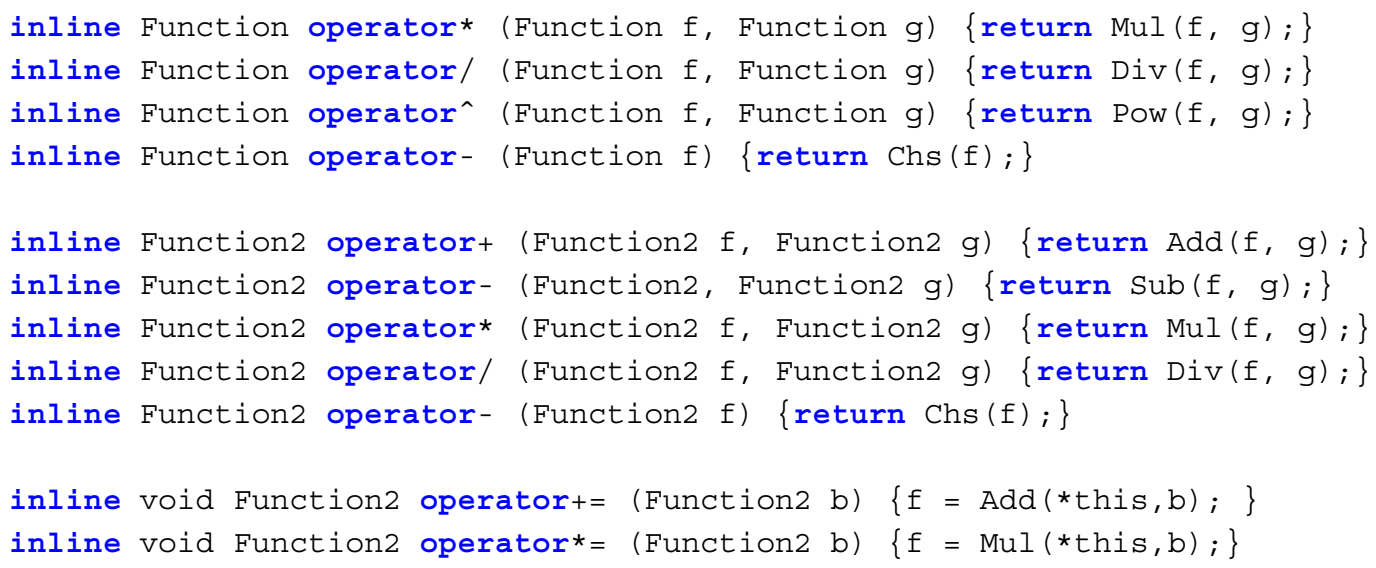

To construct effectively the classical functions, we define the class CMonome for $c x^{n}$ and the class CMonome2 for $c x^{n_{1}} y^{n_{2}}$.

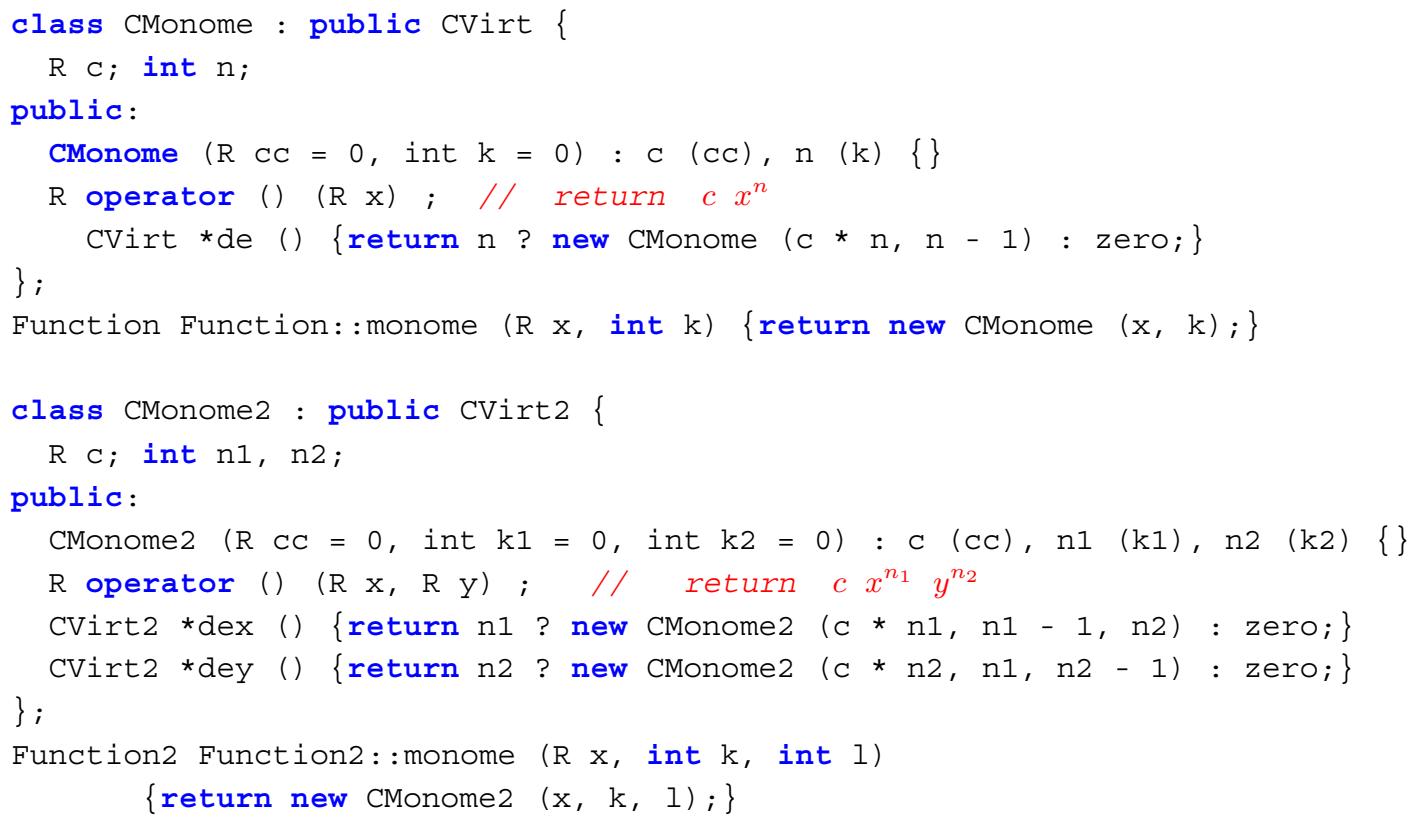

With the same technique, we construct the classes using the same rules:

CFunc a classical function defined with a pointer to a function (of type $R(*)(R)$ ). This class derives form Cvirt

CComp a class to handle the composition of $f, g$, like $(x) \rightarrow f(g(x))$

CComb a class to handle the composition of $f, g, h$, like $(x) \rightarrow f(g(x), h(x))$.

CFunc2 a classical function defined with a pointer to a function (of type $R(*)(R, R)$ ). This class derives form Cvirt2

CComp2 a class to handle the composition of $f, g$, like $(x, y) \rightarrow f(g(x, y))$

CComb2 a class to handle the composition of $f, g, h$, like $(x, y) \rightarrow f(g(x, y), h(x, y))$ 
Now to finish, we just give the definitions of the usual global functions.

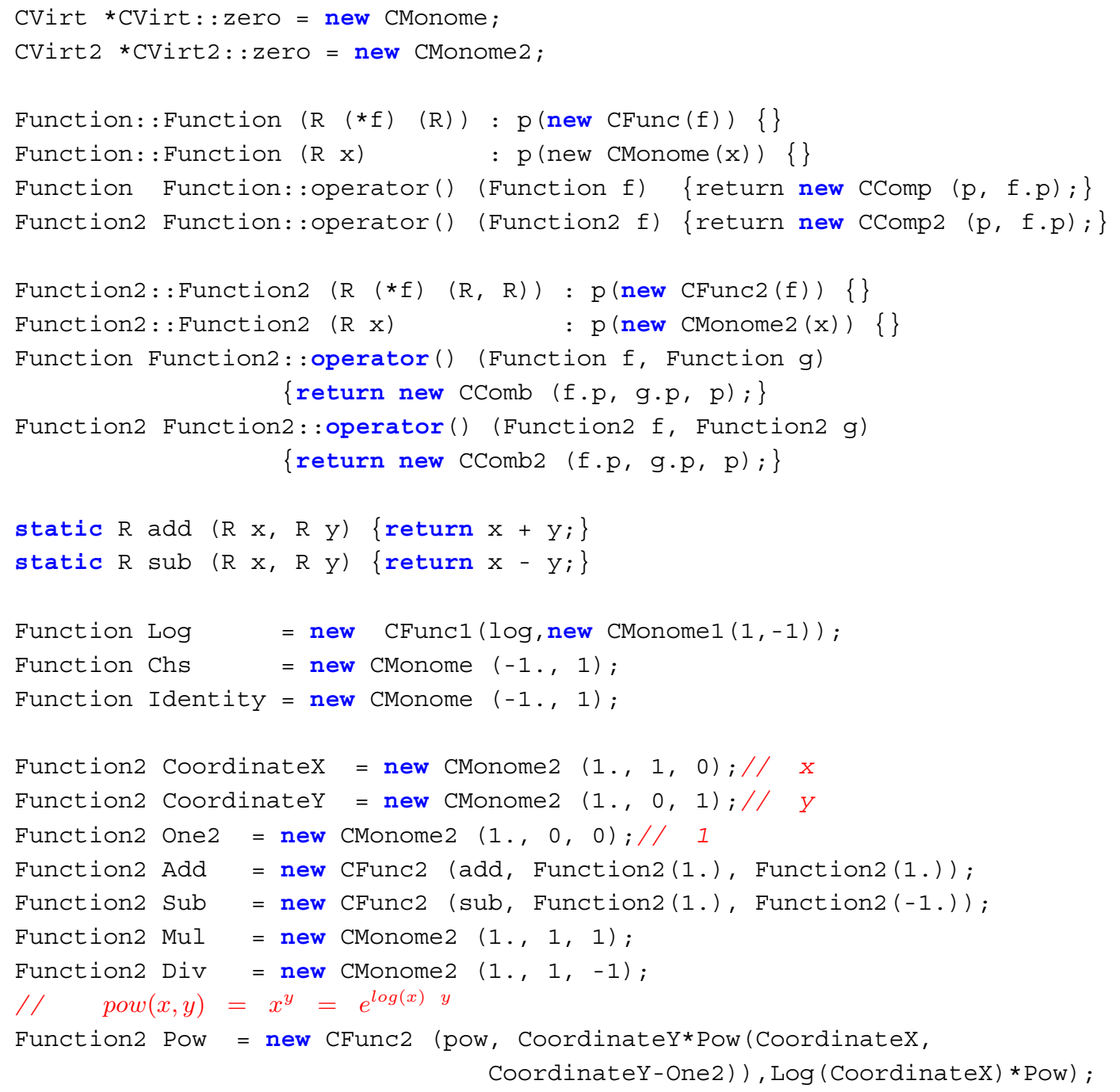

And for example to construct, the sin, cos functions, we just write:

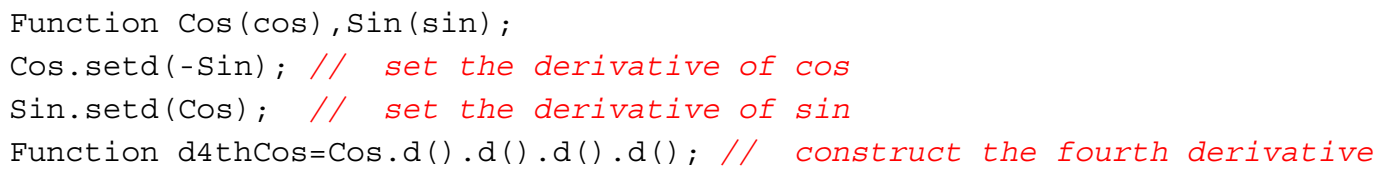

\section{Design of VARIATIONNAL FORMS}

Consider a PDE problem on a domain $\Omega$, for example, we take the Stokes problem:

Find the velocity vector $\boldsymbol{u}$ and the pressure $p$ of a fluid defined in the domain $\Omega$, such that

$$
\left.\begin{array}{rl}
\alpha \boldsymbol{u}-\nu \Delta \boldsymbol{u}+\nabla p & =0 \\
\nabla \cdot \boldsymbol{u} & =0
\end{array}\right\} \quad \text { in } \Omega
$$


For simplicity, let us choose Dirichlet boundary conditions on the velocity: $\boldsymbol{u}=\boldsymbol{u}_{\Gamma}$ on $\Gamma=\partial \Omega$. Here $\alpha$ and $\nu$ can be given positive functions ( $\alpha$ can be zero but $\nu$ must be greater than a constant number $\beta>0$.

To solve this problem, we introduce the variational form of equation (1). Take a test velocity vector $v$, null on $\Gamma$, and take a test pressure function $q$, and multiply the first equation by $v$ and integrate; do the same on the second equation with function $q$. We get:

$$
\begin{aligned}
-\int_{\Omega} \alpha \boldsymbol{u v}+(\nu \Delta \boldsymbol{u}) \boldsymbol{v}+(\nabla p) \boldsymbol{v} & =\int \boldsymbol{f} \boldsymbol{v} \\
\int_{\Omega}(\nabla \cdot u) q & =0 .
\end{aligned}
$$

After integration by parts the variational formulation of the Stokes problem is: find $\boldsymbol{u} \in H^{1}(\Omega)^{2}$ and $p \in L^{2}(\Omega)$, with $\boldsymbol{u}=\boldsymbol{u}_{\Gamma}$ on $\Gamma$, such that:

$$
\begin{aligned}
\int_{\Omega} \alpha \boldsymbol{u v}+\nu \nabla \boldsymbol{u} \cdot \nabla \boldsymbol{v}+\int \nabla p \cdot \boldsymbol{v} & =\int_{\Omega} \boldsymbol{f} \boldsymbol{v}, & & \forall \boldsymbol{v} \in H_{0}^{1}(\Omega)^{2} \\
\int_{\Omega} \nabla \cdot \boldsymbol{u} q & =0, & & \forall q \in L^{2}(\Omega) .
\end{aligned}
$$

The variational form is the sum of a bilinear form $a$, and a linear form $l$.

$$
\begin{gathered}
a((\boldsymbol{u}, p),(\boldsymbol{v}, q))=\int_{\Omega} \alpha \boldsymbol{u} \boldsymbol{v}+\nu \nabla \boldsymbol{u} \cdot \nabla \boldsymbol{v}+\int \nabla p \cdot \boldsymbol{v}+\int_{\Omega}(\nabla \cdot \boldsymbol{u}) q \\
l((\boldsymbol{v}, q))=-\int_{\Omega} \boldsymbol{f} \boldsymbol{v}=0 .
\end{gathered}
$$

The problem is: find $u \in H^{1}(\Omega)^{2}$ and $p \in L^{2}(\Omega)$, with $u=u_{\Gamma}$ on $\Gamma$, such that:

$$
a((\boldsymbol{u}, p),(\boldsymbol{v}, q))+l((\boldsymbol{v}, q))=0 \quad \forall(\boldsymbol{v}, q) \in H_{0}^{1}(\Omega)^{2} \times L^{2}(\Omega) .
$$

The bilinear form is the integral of sums of product an unknown functions or their derivative and test function or their derivative. Formally, we have in this case $n=3$ types of unknowns functions $u_{1}, u_{2}, p$ associated respectively to the number $0,1,2$, and the function unknowns $w$ can appear in three states: the function $w$ (number 0$), \partial_{x} w$ (number 1), or $\partial_{y} w$ (number 2). We can do exactly the same numbering for the test functions part. So the bilinear form can be written as a sum of terms in $\mathcal{C}^{0} \times[\{0, . ., n-1\} \times\{0,1,2\}] \times[\{0, . ., n-1\} \times\{0,1,2\}]$ where $\mathcal{C}^{0}$ is the set of functions, $n$ is the number of unknown functions, the first $[\{0, . ., n-1\} \times\{0,1,2\}]$ term define the unknowns and the last $[\{0, . ., n-1\} \times\{0,1,2\}]$ term define the test function part.

For example the terms of the bilinear part of the variational form can be translated in:

$$
\begin{array}{clc}
\alpha u v & \equiv & (\alpha,[0,0],[0,0])+(\alpha,[1,0],[1,0]) \\
\nu \nabla u \cdot \nabla v & \equiv+(\nu,[0,1],[0,1])+(\nu,[0,2],[0,2])+(\nu,[1,1],[1,1])+(\nu,[1,2],[1,2]) \\
\nabla p \cdot v & \equiv & (1 .,[2,1],[0,0])+(1 .,[2,2],[1,0]) \\
\nabla . u q & \equiv & (1 .,[0,1],[2,0])+(1 .,[1,2],[2,0]) .
\end{array}
$$

We can do the same analysis for the linear form, and to close the analysis we have just to remark that in general we have only two types of integrals: over the domain or on its boundary.

To make the implementation easier we introduce, a kind of linear combination, or a sparse vector of $R^{(I)}$ like:

$$
\sum_{k=1}^{n} r_{k} i_{k}, \quad \forall k=1 . . n, r_{k} \in R \text { and } i_{k} \in I .
$$


Where $R$ here, is an algebra of functions, and where $I$ is the set of indices (in case of linear form an index is just a pairs of integers).

A simple member function add $(r, i)$ adds a term $(r, i)$ to the linear combination, plus similarly the classical operator + and external product $*$ of $R^{(I)}$.

Thanks to the STL the class LinearComb is easy to constuct with vector and pair where a pair is just the following structure:

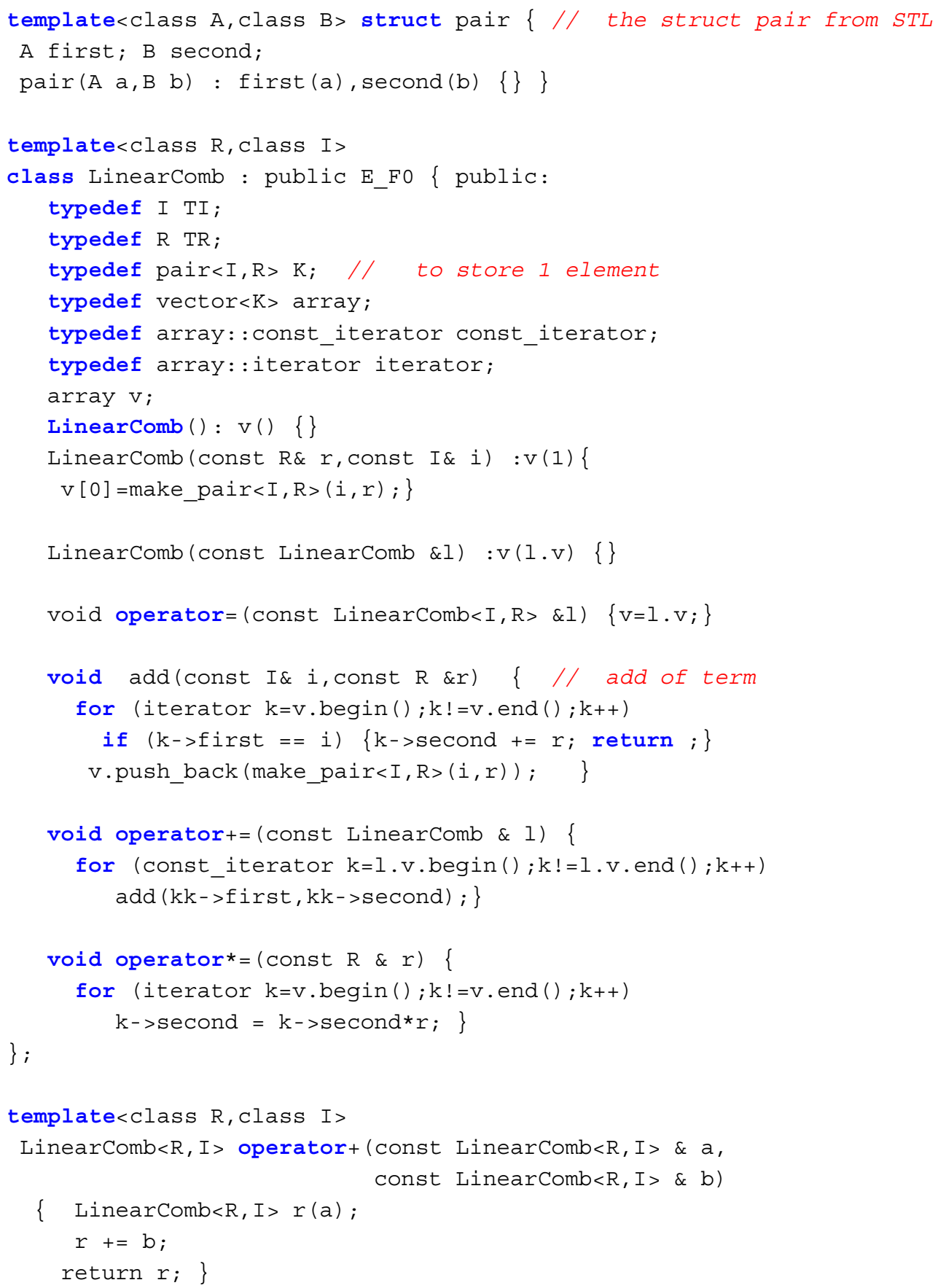




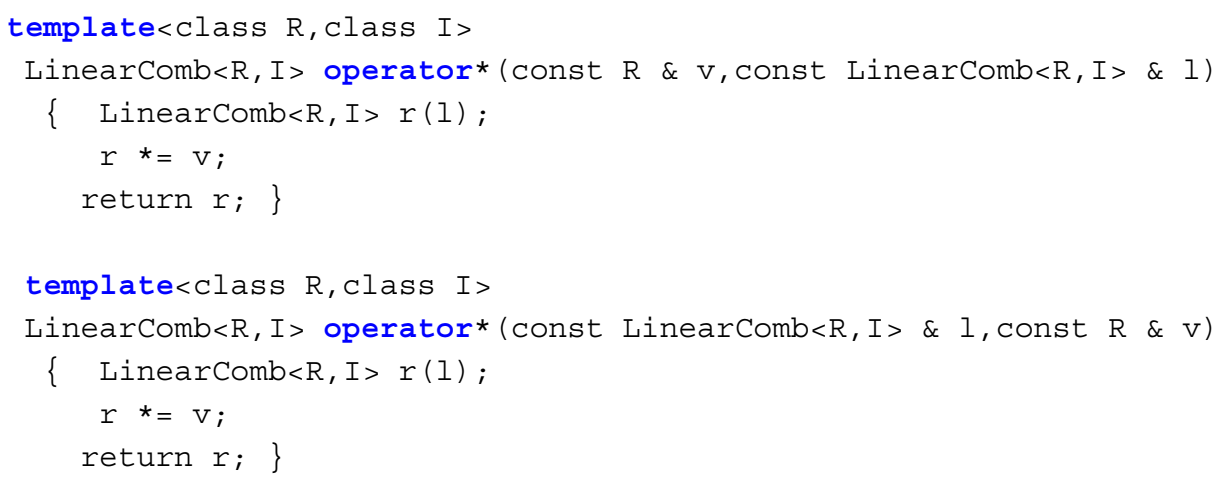

with:

enum operatortype $\left\{o p \_i d=0, o p \_d x=1, o p \_d y=2, o p \_d z=3\right\}$;

template <bool testfunction>

struct FunctionNumber \{

const int $i$;

FunctionNumber (int $j): i(j)\{\}$

operator int const () const \{return $i ;\}$

\} ;

void operator $=($ int $j) \quad\{i=j i\}$

The $\mathrm{C}++$ types corresponding to the two kinds of linear variational forms are:

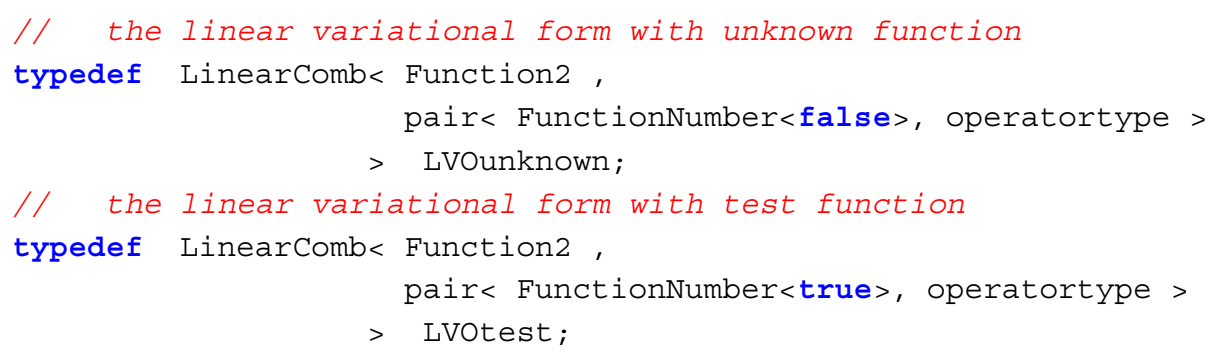

The $\mathrm{C}++$ types to handle bilinear forms is:

typedef LinearComb $<$ Function2,

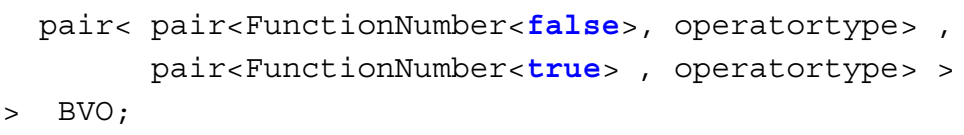

A BVO is a construct with a product of LVOtest and LVOunknown. We have used the two following product operators:

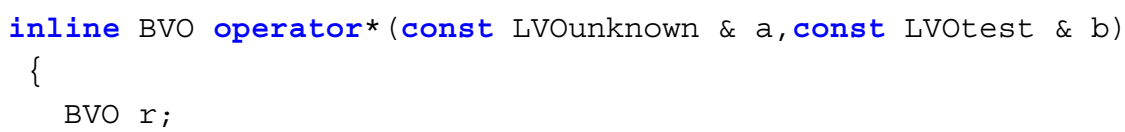




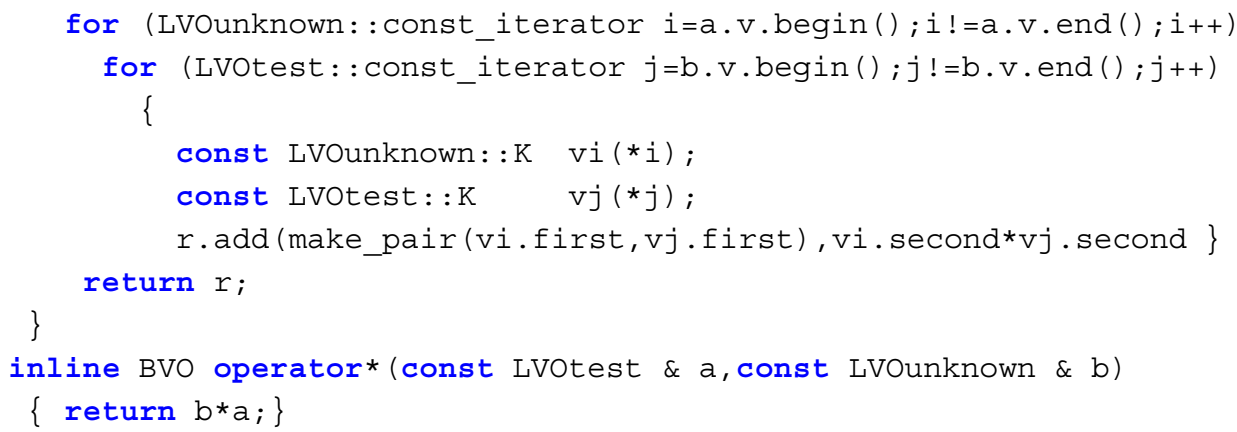

The function to differentiate a linear combination is:

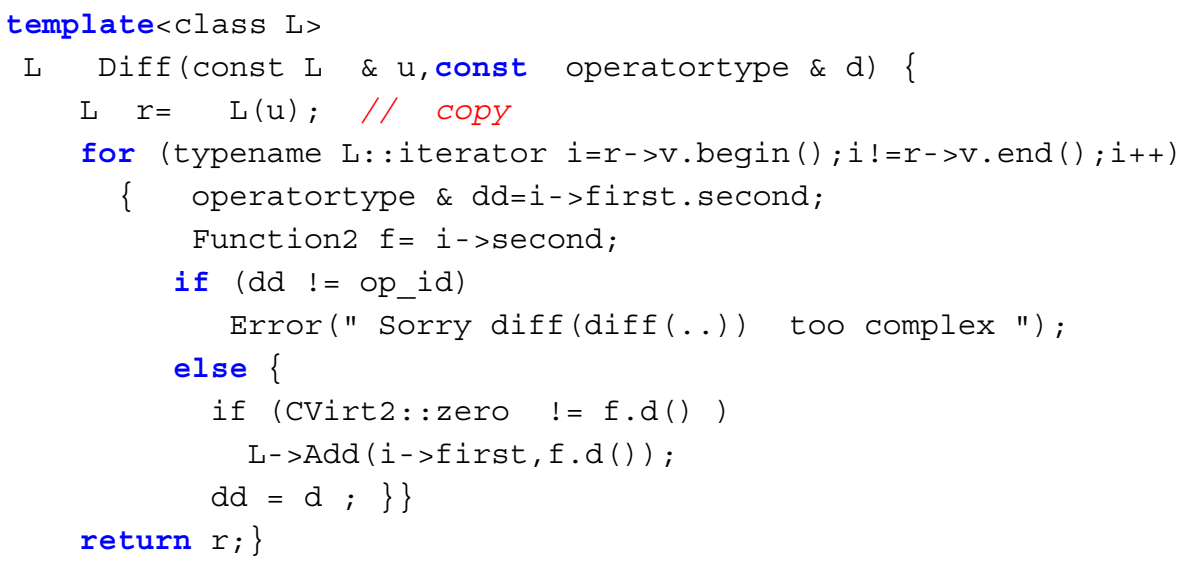

The variational forms for test and unknown functions are:

inline LVOtest test(int i) \{return LVOtest(i,op_id);

inline LVOunknown unknown(int i) \{return LVOunknown(i, op_id);

and the two functions $\mathrm{dx}=\partial_{x}$ and $\mathrm{dy}=\partial_{y}$ to differentiate are:

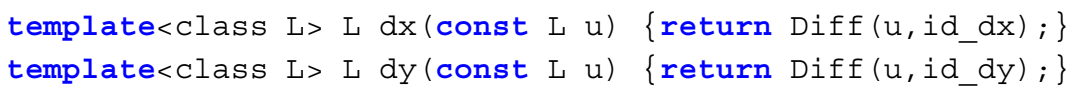

Now we use all the classes to construct the variational form of the Stokes equation. First, construct all the linear forms corresponding to the unknown functions $u 1, u 2, p$ and the corresponding to the test functions $v 1, v 2, q$ :

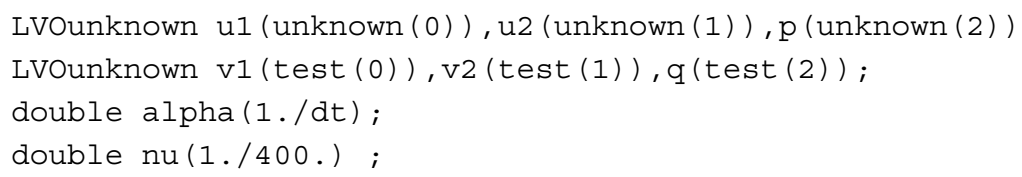

The translation of the bilinear form (3) and the linear form (4) are:

BVO $\mathrm{a}=(\mathrm{u} 1 * \mathrm{v} 1+\mathrm{u} 2 * \mathrm{v} 2) * \mathrm{alpha}$

$$
+(\mathrm{dx}(\mathrm{u} 1) * d \mathrm{x}(\mathrm{v} 1)+\mathrm{dy}(\mathrm{u} 1) * d \mathrm{y}(\mathrm{v} 1)
$$


LVOtest $1=\mathrm{v} 1 * \mathrm{f} 1+\mathrm{V} 2 * \mathrm{f} 2$;

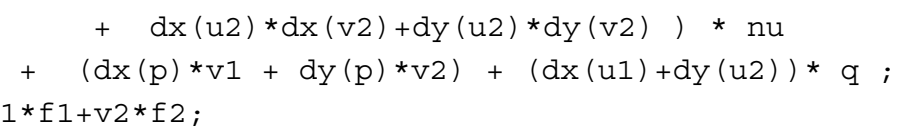

As we can see, this writing is very close to the mathematical formulation.

To compute the elementary matrix $a$ on an element $K$, we can use this kind of lines:

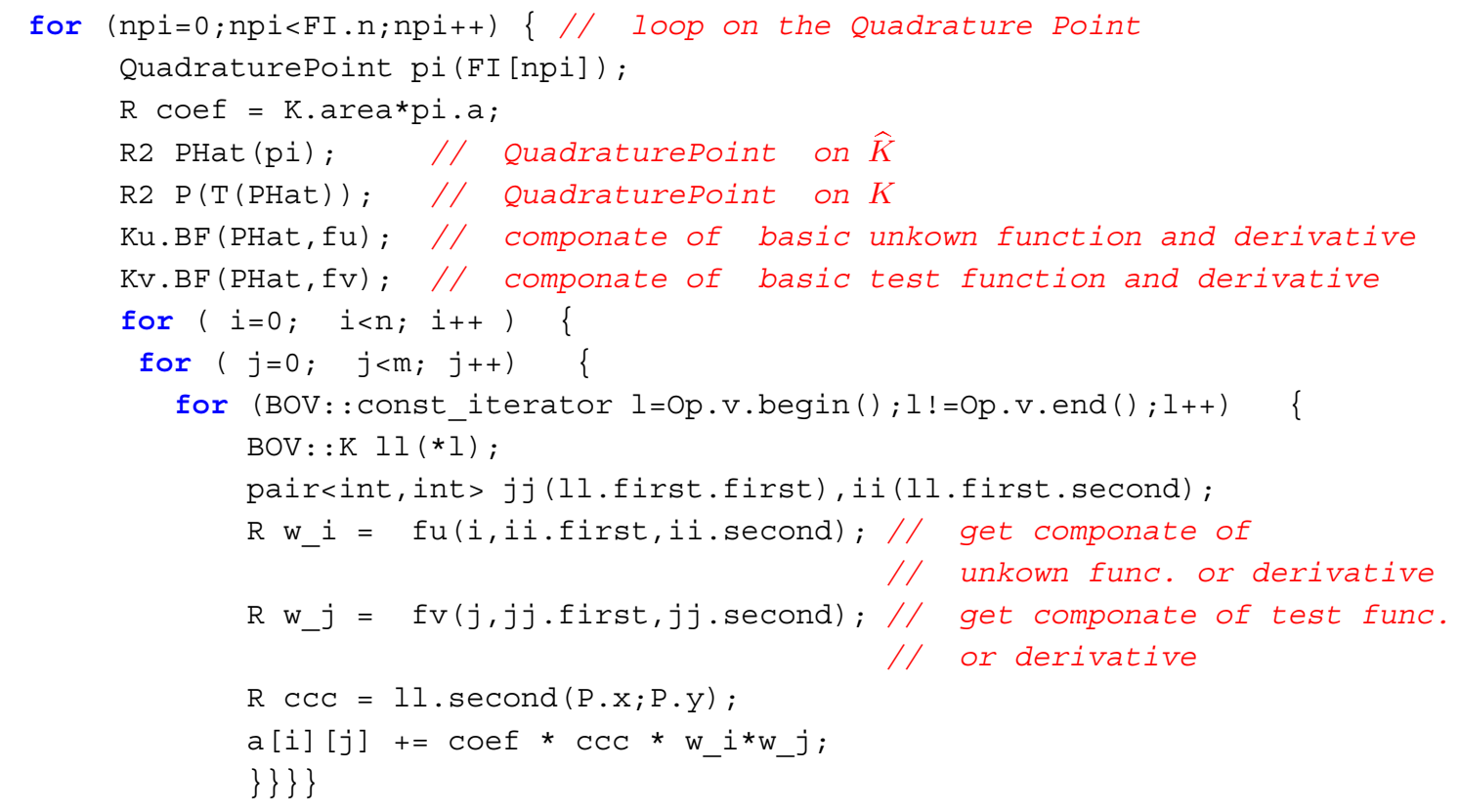

where $K u$, and $K v$ are the objets associed the finite element space on the element $K$.

\section{Designing a multitype Virtual machine}

In this section, we explain the design of the virtual machine for the language. This language can handle lots of types, as can be seen in Section 4.1; it is relatively easy to handle functions with the virtual mechanism. As we remark previously, the virtual machine is based on an extended algebra of "instruction function" with one parameter the stack memory. The stack memory is used to store all the local variable of the language. The "instruction function" can return any type of data. We have two kinds of data the small ones (example: bool, long, double, complex) and the large ones (example: string, Mesh, ...), classically the small data are stored directly, and the large ones are stored via a pointer.

The class basicForEachType stores all the data of language's type.

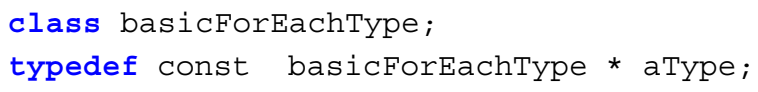

The size of all small types must be smaller than 24 .

typedef unsigned char AnyData[24];

To make the link between the name of the type and the user language type (a pointer to a basicForE- 
achType), we use the run time type interface (RTTI) of $\mathrm{C}++$. So to get the user language type we use a map of the STL map_type:

extern map<const string,basicForEachType * map_type; file.

We can't use directly the pointer of the typeid $(T)$ because the data storage depends on the compilation

The following template function atype $<T>()$ returns the pointer to the internal type.

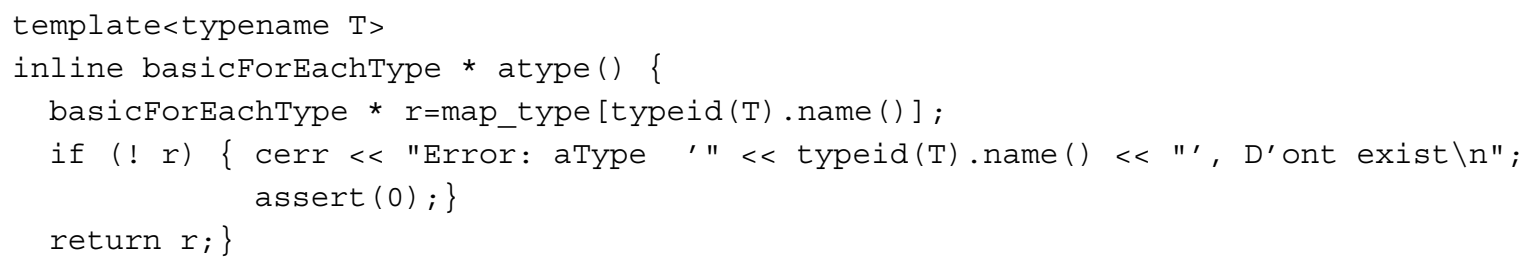

The class to store every small data or pointer with or without dynamic type checking depends on the preprocessor macro WITHCHECK:

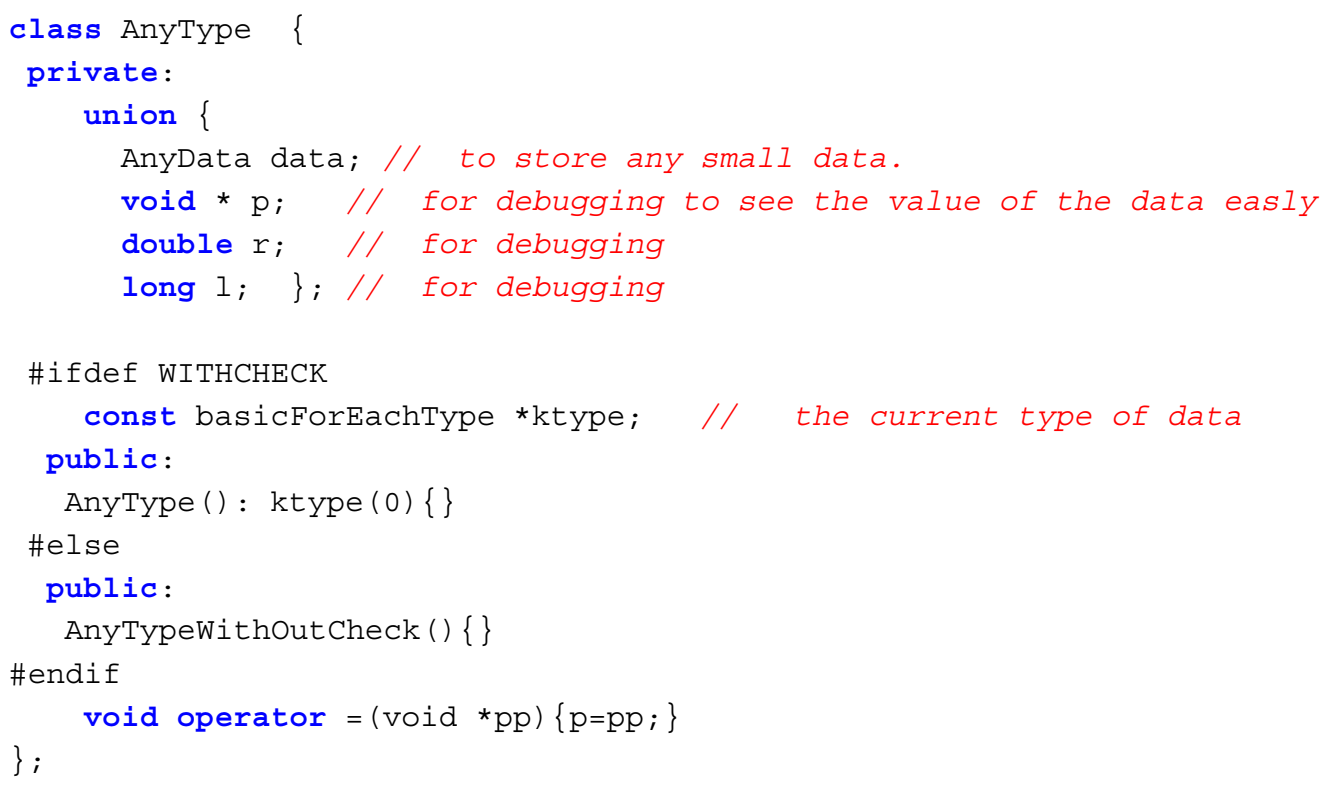

This is because compilers do not work generally with template constructors and template cast operators, we use two template functions AnyType SetAny $<\mathrm{T}>\left(\begin{array}{ll}\mathrm{T} & \&\end{array}\right)$ and $\mathrm{T}$ GetAnY $<\mathrm{T}>($ AnyType \&) to transform back and forth beetween a $\mathrm{T}$ and an AnyType.

First, we introduce, a small class CheckSize just to perform size checking at $\mathrm{C}++$ compilation time. We use a specialization of this class with a Boolean ok. If this Boolean is false then we use a private constructor to generate a compile error.

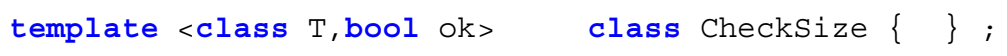




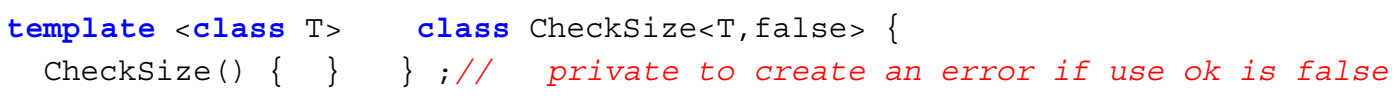

Now, the function SetAny transforms a type $\mathrm{T}$ to AnyType with a bit copy:

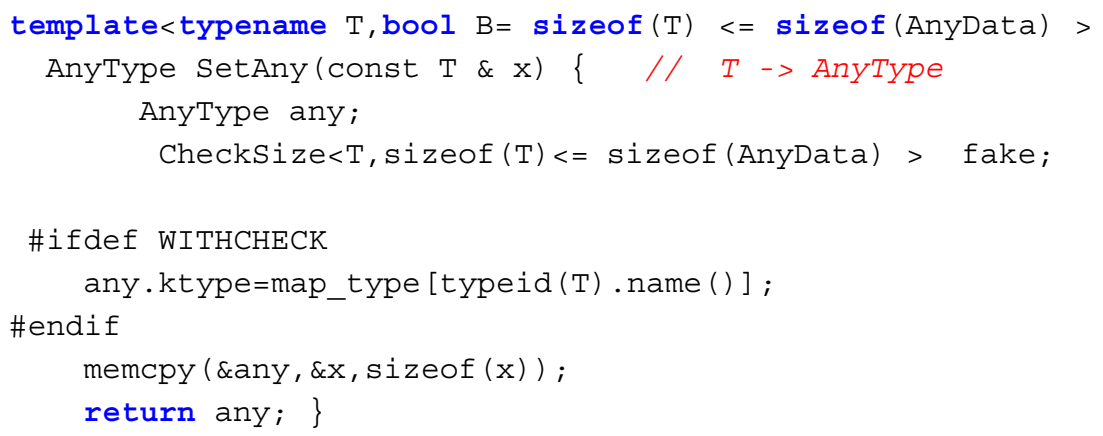

Similary, you can write the following functions

template<typename $\mathrm{T}>\mathrm{T}$ GetAny (AnyType \& );

AnyType PtrtoAny (PtrtoAny (void * p, const basicForEachType * $r$ );

We can use, an extend algebra of function to model the instructions of the language. The parameter of the function has to be the stack if we want to product a reentrant code. In the stack, we store all the local variables of the procedure. Secondly, we have to construct a class basicForEachType to define all the types of the user language.

\section{typedef void *Stack;}

So an instruction of the language is an Expression, and an Expression is a pointer to the class E_F0.

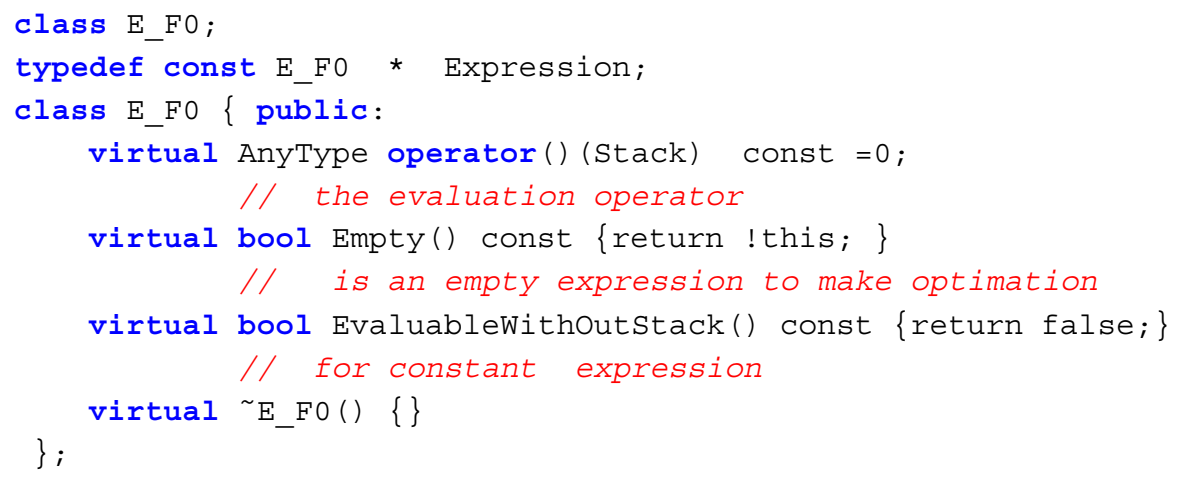

We use exactly the same technique as in the algebra of functions to construct the virtual machine. Before getting a value of type $\mathrm{Tg}$ we use the GetAny<Tg> () function and to return a value of type Ts, we use the SetAny $<\mathrm{Tg}>$ ( ) function. 
For example, a local variable of the language can be

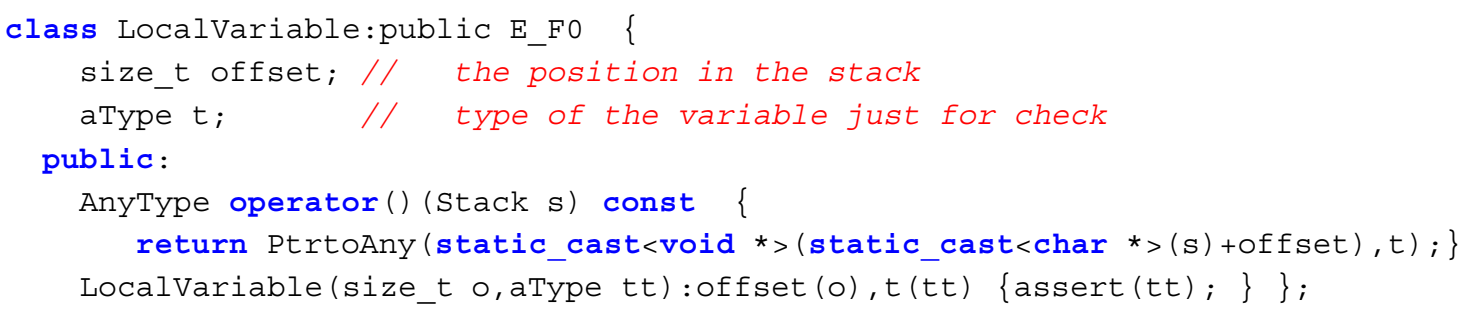

It is very simple to write an expression to make a "for loop" with the $\mathrm{C}++$ syntax. The return type of a "for loop" expression is clearly a void. The break and the continue instruction are handled by the C++ exception mechanism. So the class is:

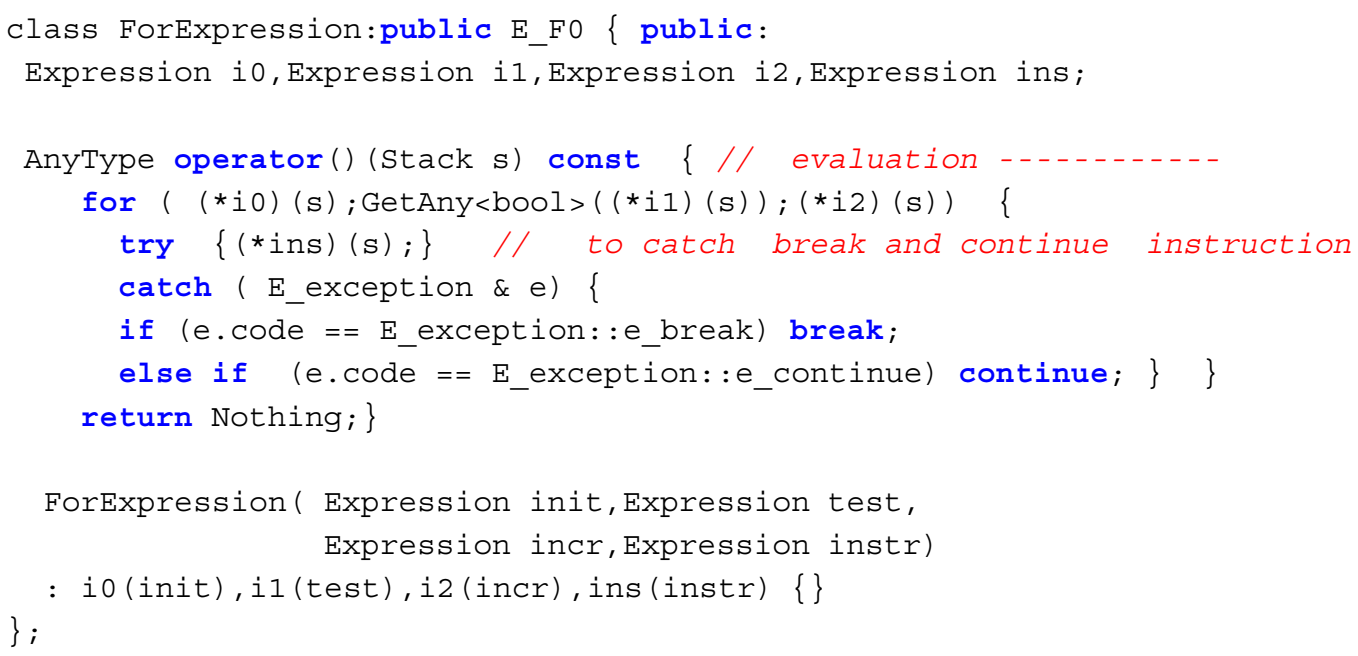

At compilation time, the type of the expression must be known, so we need to define what is the type of the objects of the languages.

Polymorphism for operators and functions is easy to achieve. First, an operator is just a function with a special name ${ }^{3}$ and second we just choose the function to call with the type of parameters. But unfortunately, in the language we have four scalar types bool, long, double, complex<double >. We want to be able to add every type to every type, so $4 * 2$ cases. A lot of operators, the risk of mistake is too important. We have no easy way to do that with templates (an operator parameter); so to solve this problem, we can use automatic casting like in $\mathrm{C}++$. It is clear that one can cast (promote) a numeric type to another type, e.g. a float into a complex. Now we have to solve the problem of ambiguity ( (long) + (double)) could mean (long) + (long), (double) + (double), (complex) + (complex), or (string) + (string). The rule to solve this kind of ambiguity is to choose in order to minimize the number of casts (one here), and we add a level of a priority only for binary operator to remove the last ambiguity beetween ((long) + (long)) and ((double) + (double)) and choose (double) + (double), where the level is 10,20,30,40,50 for respectively bool, long, double, complex, string and the level is 0 otherwise.

In the language, we have also some "left expression" like in $\mathrm{C}++$, but expression a can be both "left expression" (in $\mathrm{a}=10$ ) and "right expression (in $\mathrm{a}+10$ ). In a "left expression", we need the memory address of the objets so the expression returns a "kind of pointer" to the objet, but otherwise in the "right expression"

\footnotetext{
3 the operator $x+y$, can be seen as the function $+(x, y)$.
} 
the expression haves to return the objets in self. To solve, this difficulty, a "kind of pointer" can be a pointer to the value or the value in self, we use the user language cast mechanism by adding one more cast (pointer to pointer value) and this kind of cast is clearly not counted when we solve the problem of ambiguity.

This is the list of the kernel type names:

Oneoperator is a class for a function name with different type of parameter;

basicForEachType is a class for a type the objets of the language;

E_FO is a pure virtual class for the instruction expression;

aType is a pointer to a constant basicForEachType;

Expression is a pointer to a class E_FO;

Function1 is the type name typedef AnyType (* Function1) (Stack, const AnyType \&);

Polymorphic is a class, which models a polymorphic object. It's derived from an E_FO;

C_FO is a class for compilation expressions, just 2 pointers. One is an Expression and the other is an aType; These two pointers define the expression and the type of the expression;

basicAC_FO is a class for an array of compilation expressions. This array is used to pass the arguments of an operator;

TableofIdentifier is a class for a table of identifiers.

\subsection{The types of the language}

The type definition is the kernel of the language, everything is in the class basicForEachType.

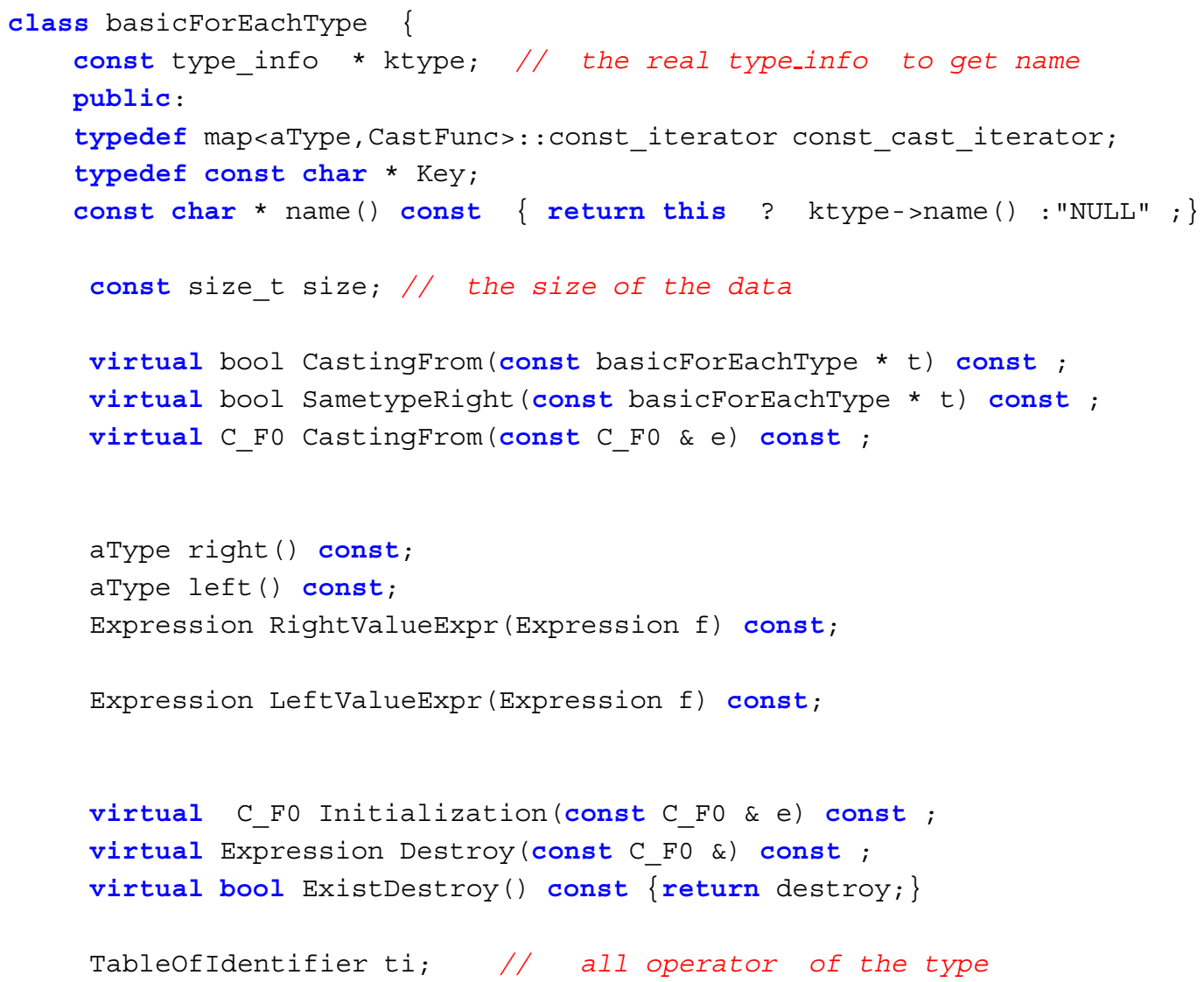




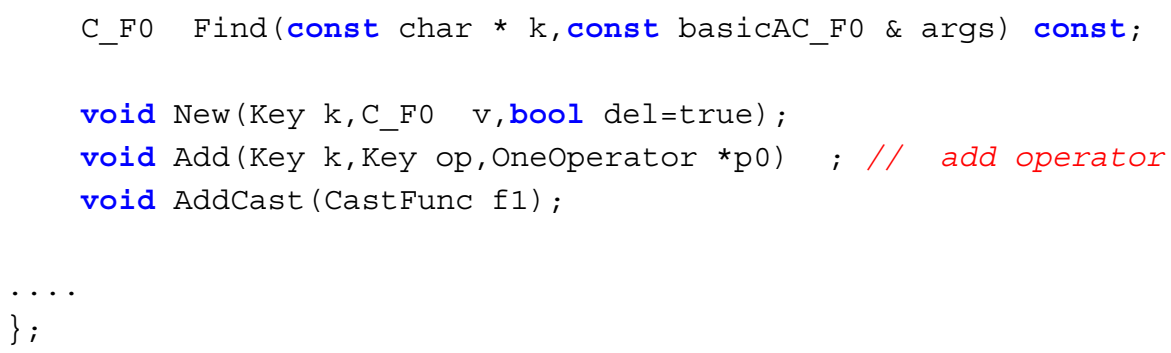

Trick: To make the automatic cast easily, we need for all the types a way to promote in this type, see the method CastingFrom to know if this possible or not to create the cast expression.

\subsection{One operator}

First we use the class Oneoperator to construct the compiler expression for one named operator in function of the type of the arguments. We have one kind of construction, and two kinds of query possible to do this operation with or without casting of the parameter. Remark that in the two cases the promotion of a left expression to a right expression is done.

In fact, we just use a Oneoperator to define the cast into this type.

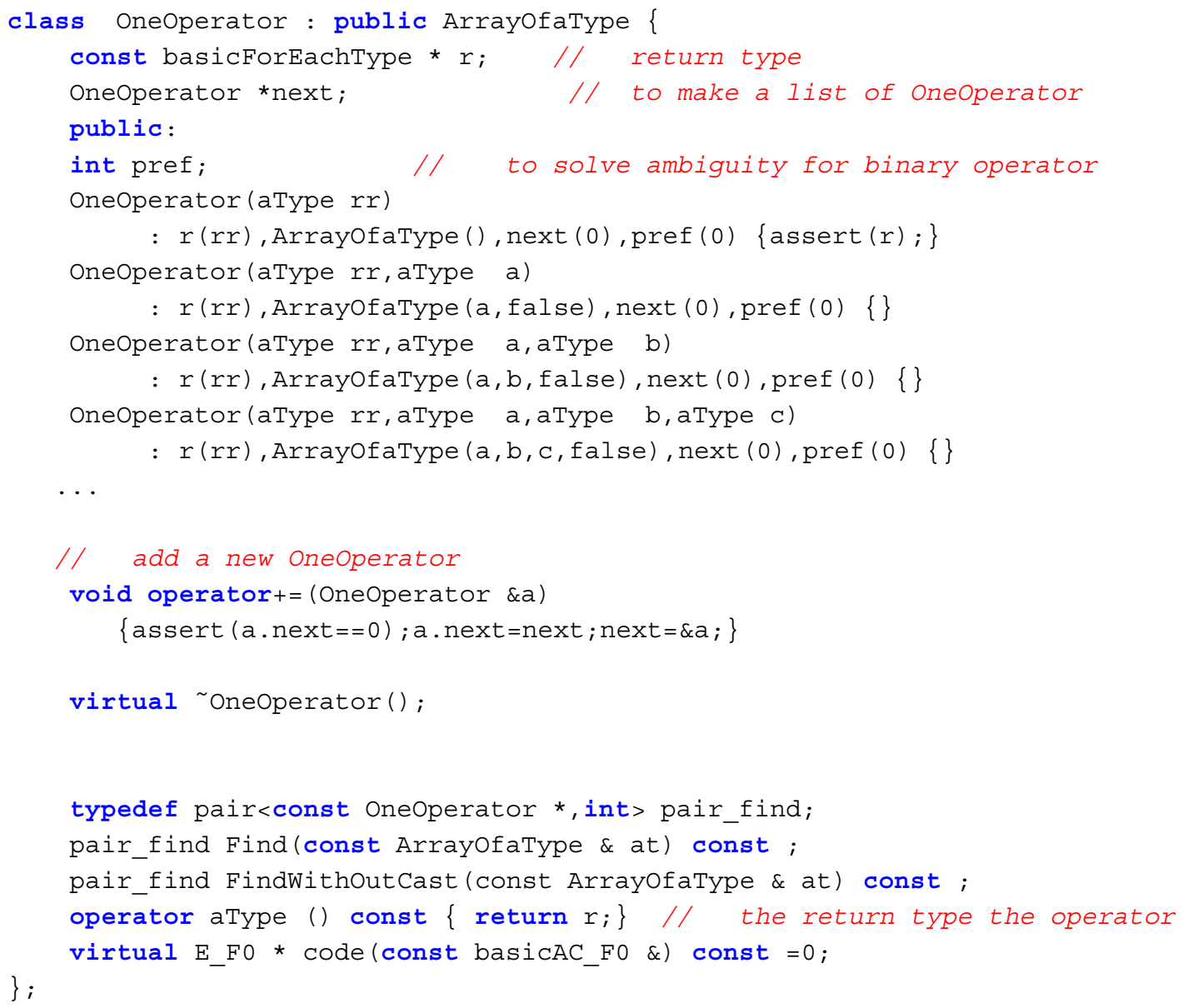


where the class ArrayofaType is just like an array of aType with a basic constructor, and a Boolean to take into account the variable parameter list if any.

The virtual function code constructs effectively the expression (a pointer to E_FO), and the two functions Find and FindWithoutCast return a pair of the Oneoperator pointer and the number of ambiguities.

\subsection{How to handle a polymorphic object}

A polymorphic object is just like a $\mathrm{C}++$ class. On a polymorphic object, we can define any operator, function, member, and method. The operator, member, method or function are defined with a string (const char *). So it is almost a map of string and Oneoperator object.

First we define a simple struct to sort in lexical order the map

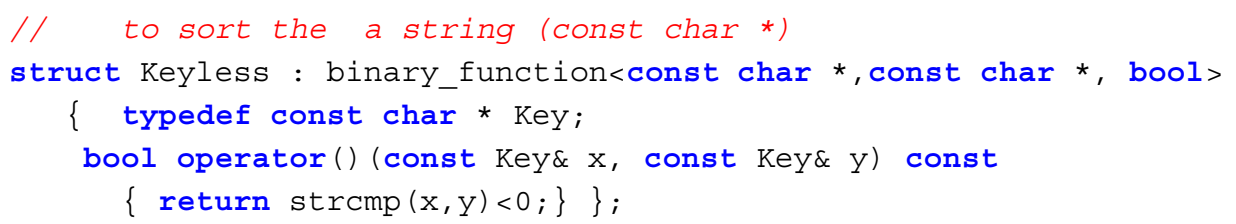

The class Polymorphic can be:

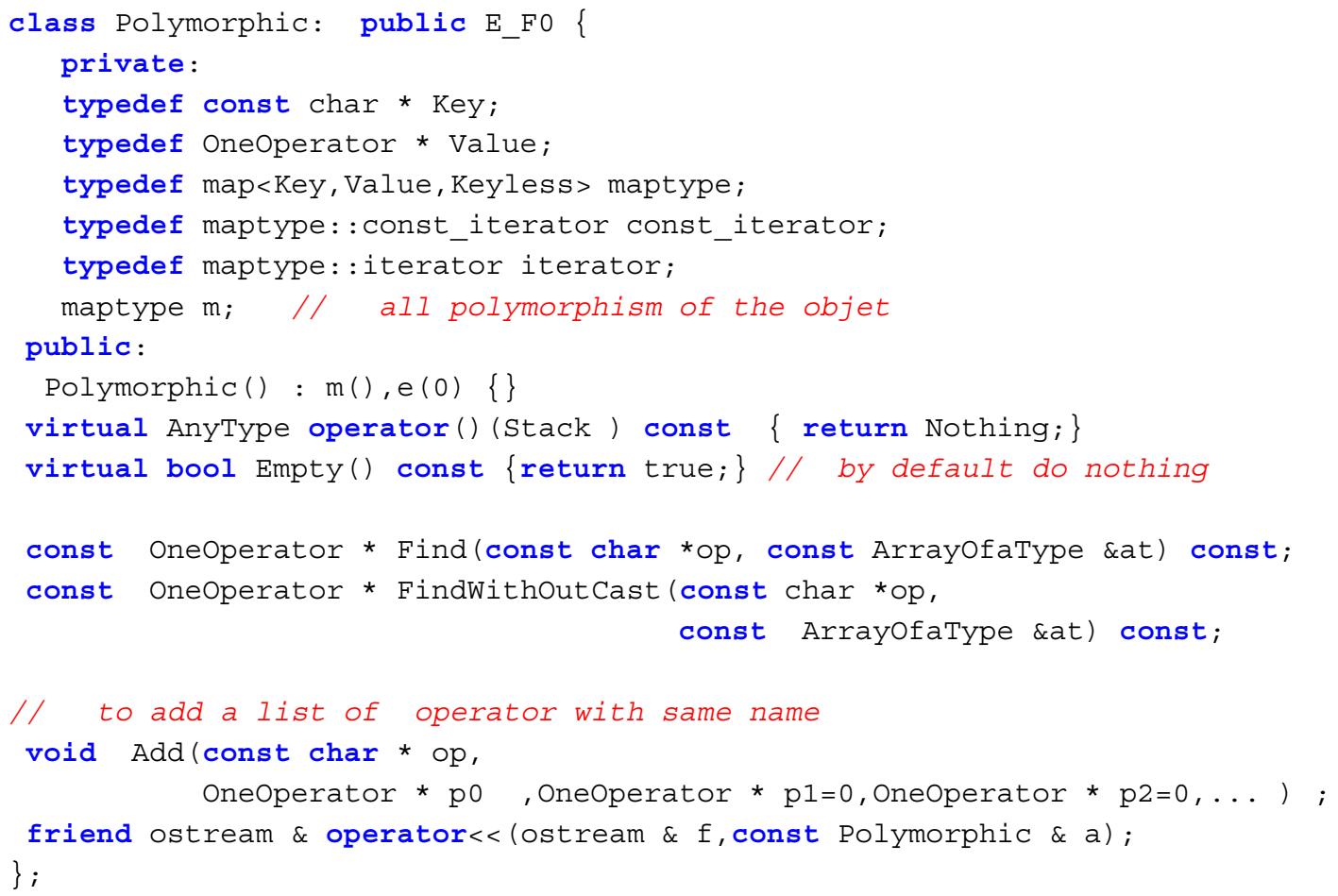

The two functions Find and FindWithoutCast are just the encapsulation of the same function of the class Oneoperator, if the operator name exists, and generate an error otherwise.

\subsection{Identifier tables}

The table of Identifier is theoretically just a STL map of names and compiled expression, but when we go outside a block we must delete all local identifier in a right order variable; so we create a new structure Value 
which derive from the class C_FO, and we add a link to the previous variable, and a Boolean if the variable must be removed.

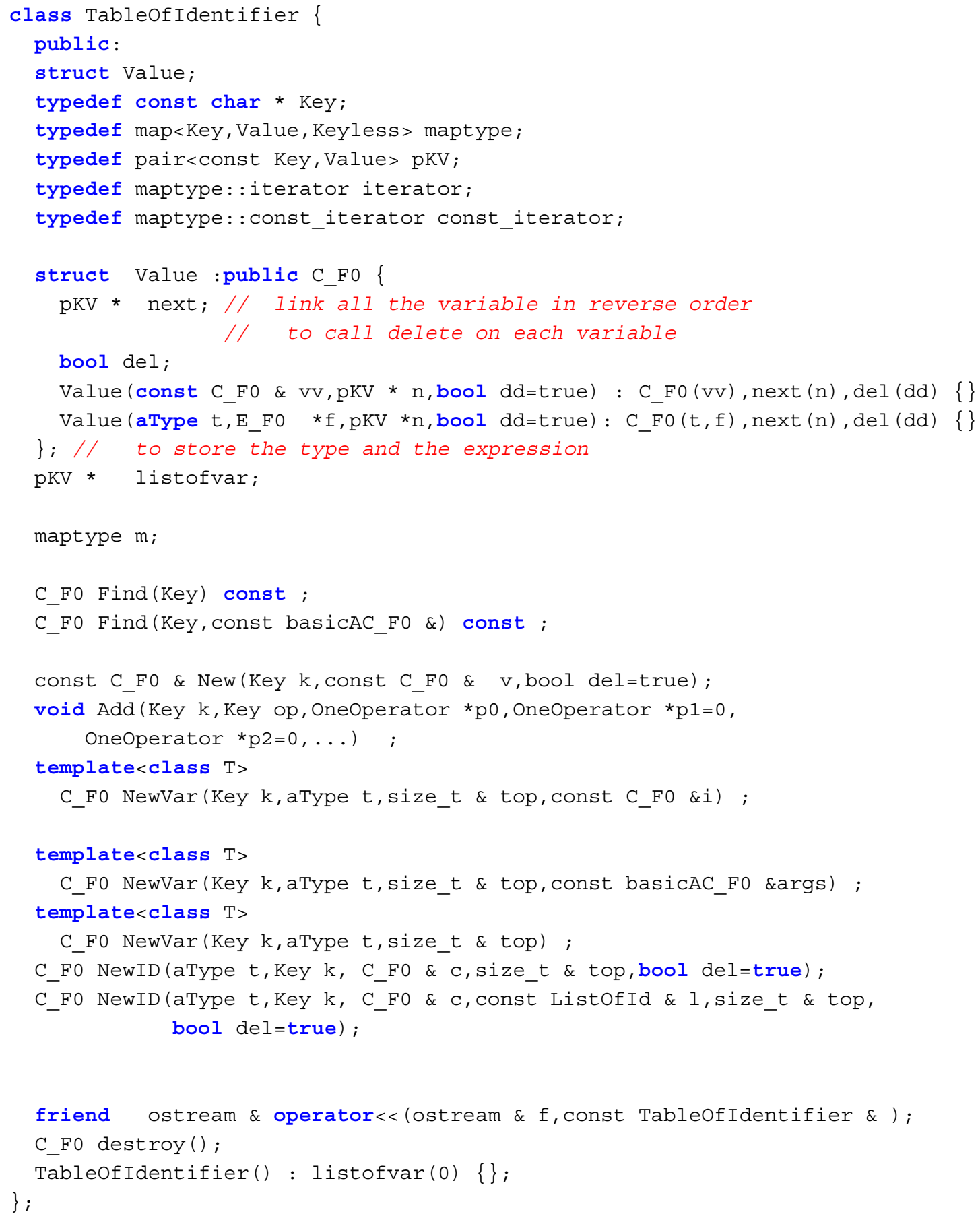




\subsection{How to use}

Finally, all the operators are in a global variable:

\section{Polymorphic * Theoperators=new Polymorphic();}

And all the globals of the language are stored in a global table of identifiers.

TableofIdentifier Global;

To add operator the + operator, we just write:

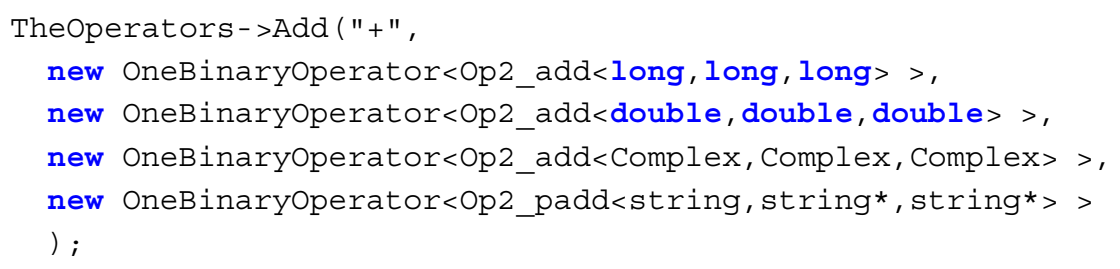

where the class OneBinaryoperator is:

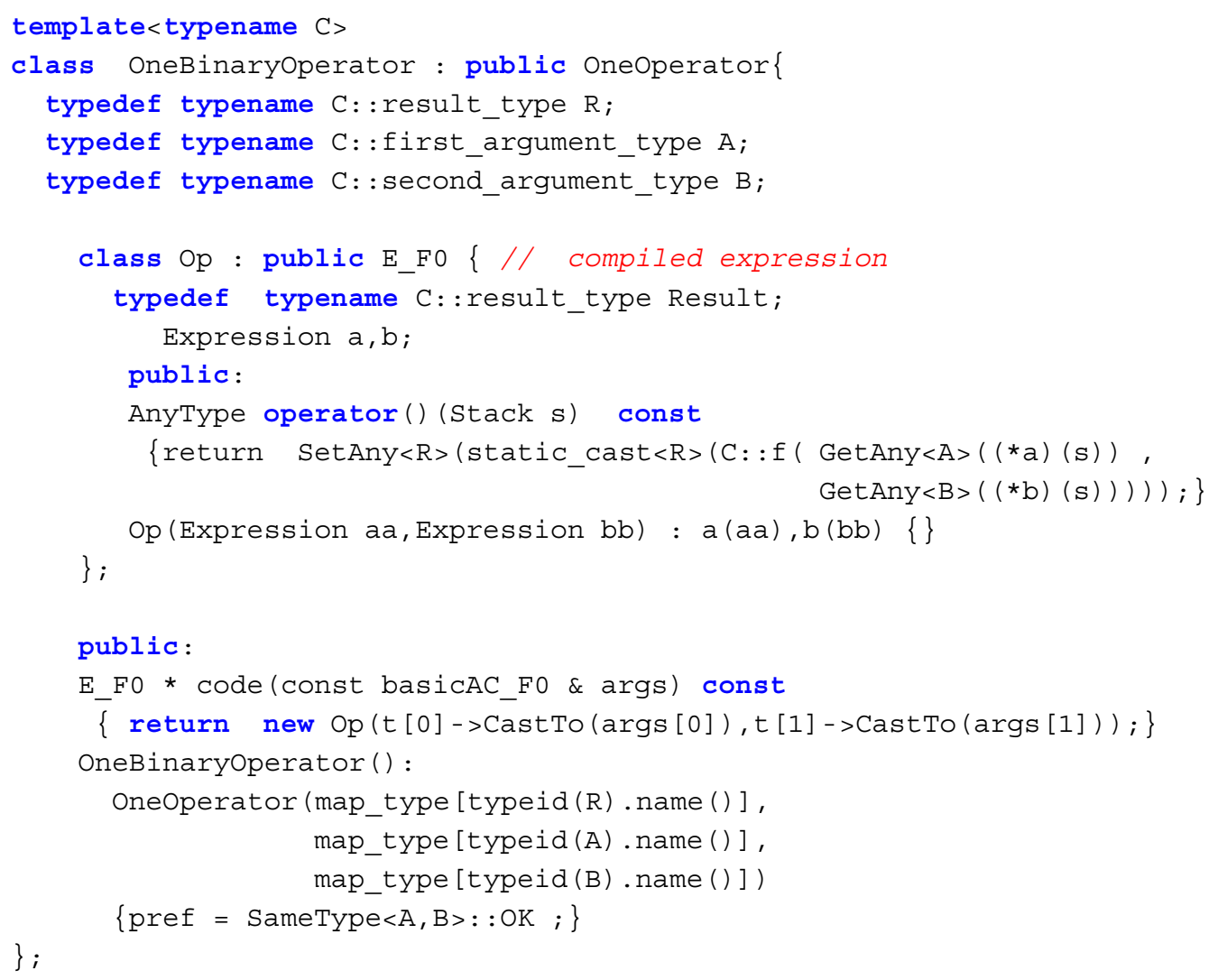


The class Op2_add to make the "add" is just:

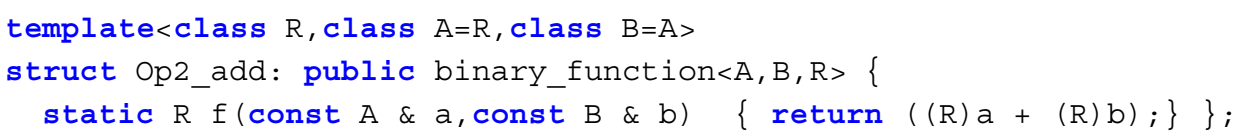

We describe the class $C_{-} F O$ and how to construct an instruction of the language.

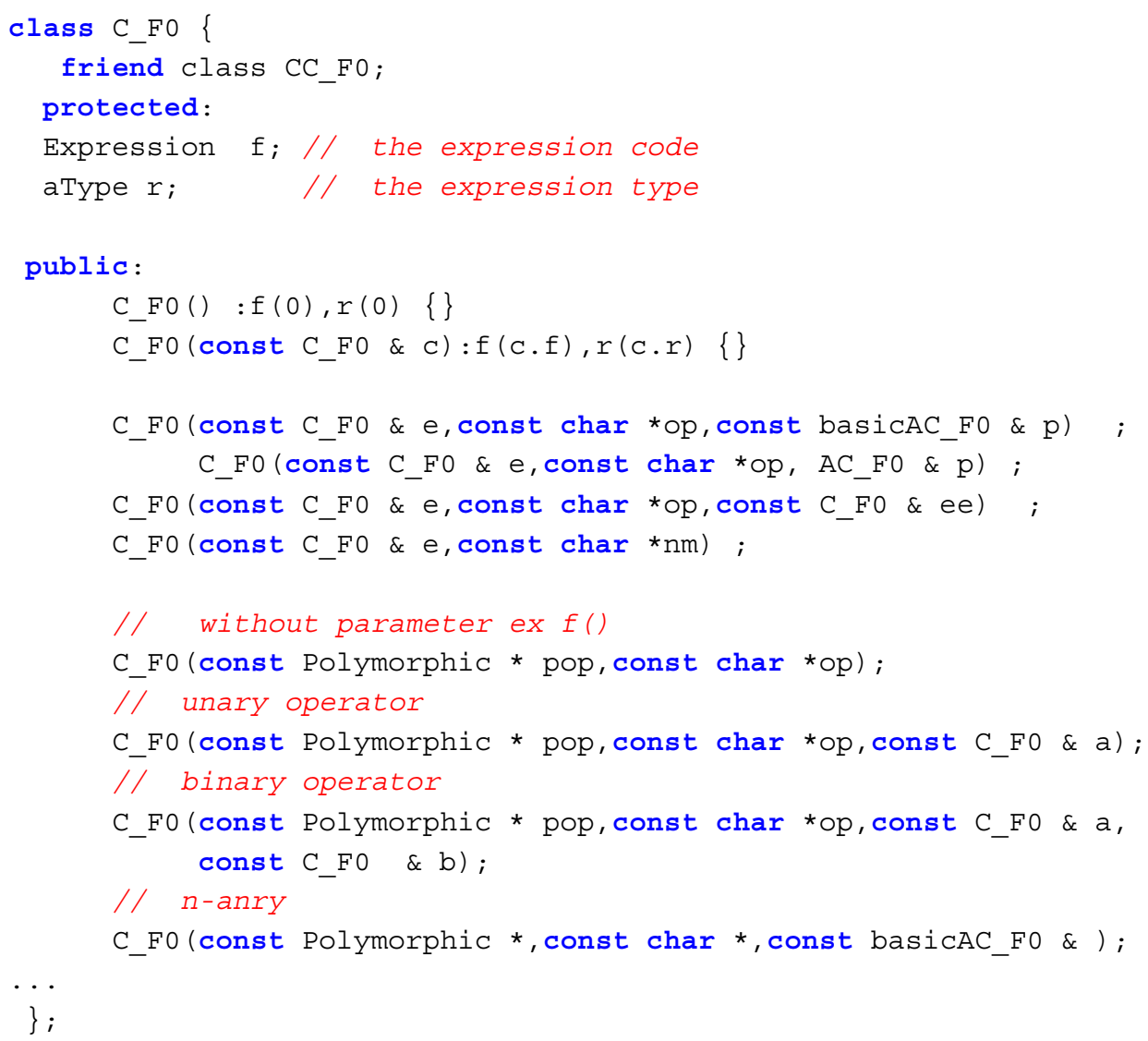

For example, to construct the expression ab sum of two expression $\mathrm{a}$, b, we have to write:

C_FO ab (Theoperators, "+", a, b) ;

We used the same rule for all the operators.

\section{BISON GRAMMAR}

Now we explain, briefly, how to use this class with the compiler bison or yacc processor [3].

First, we introduce the return type of all the terminal and non-terminal terms of the grammar. But unfortunately, it is impossible to add class with constructor in an union, so we construct another class CC_FO with the same data members $r, f$, with the assignment operator form a class C_FO, and the cast operator to class C_F0. 


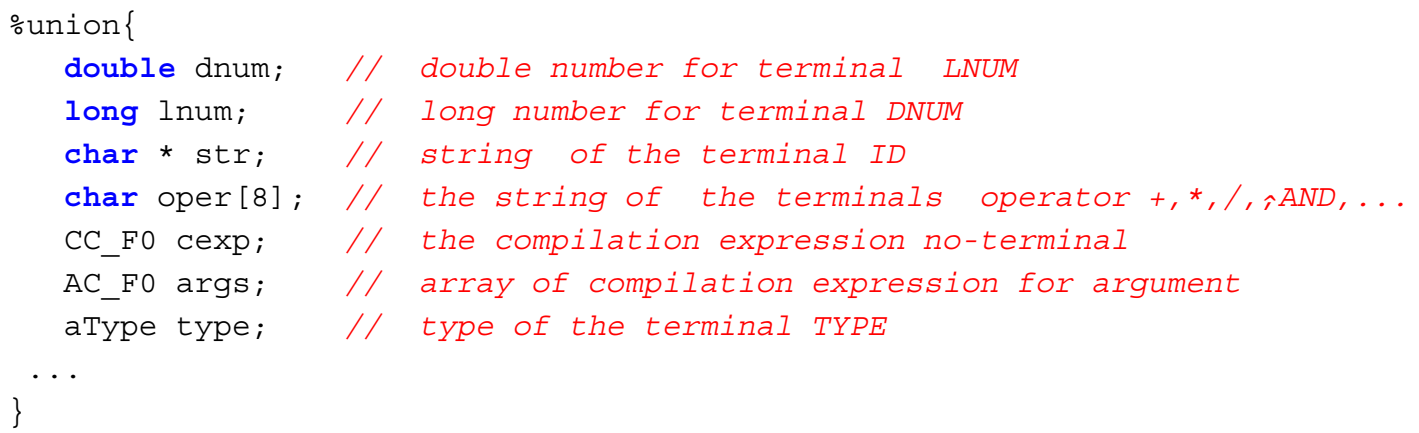

After remembering the following construction:

C_FO ab (Theoperators, "+", a, b) ;

build the sum of two expression a,b. The non terminals for "expression" with non set operator are:

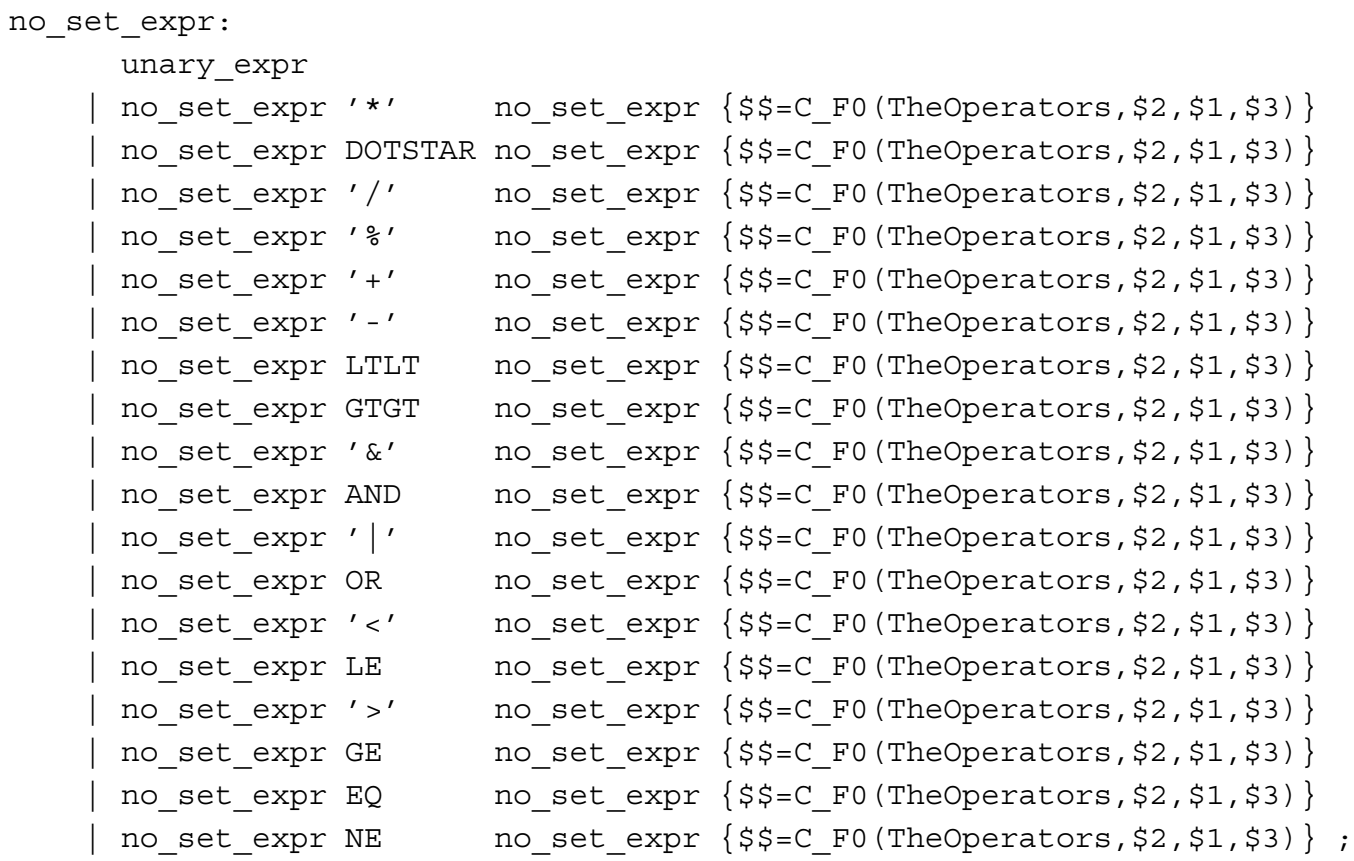

where the non terminal unary_exp handles all the left unary expressions.

An interesting part is the primary terms of the expression. This is defined with:

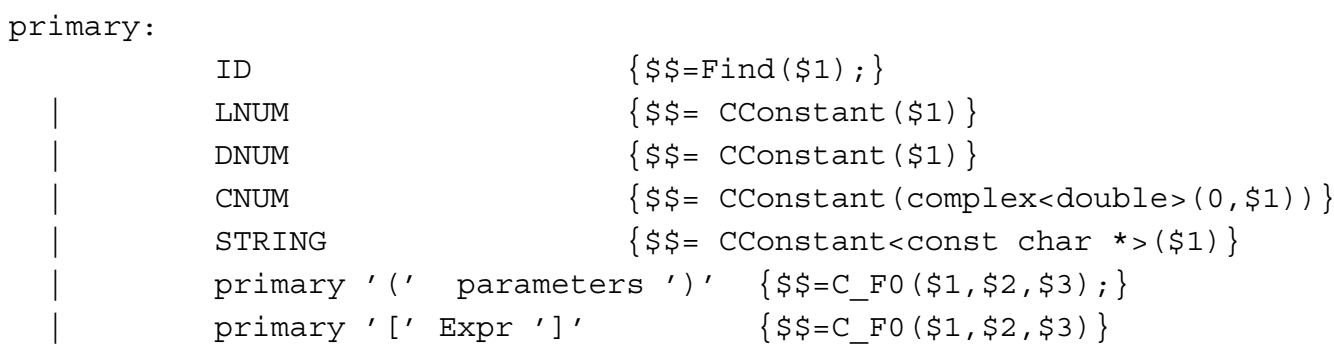




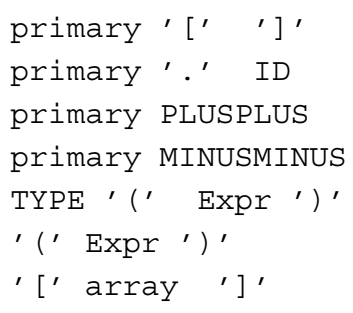

where ID, LNUM, DNUM, CNUM, STRING, TYPE, PLUSPLUS, MINUSMINUS are terminal associated to an identicator, a numeric number, a type,,++-- .

The unary operator right ++ (resp. --) is associated to the string right++ (resp. right--), just to differentiate the left or right unary operator ++ or -- .

\section{Examples with FreeFem ++}

\subsection{Navier-Stokes}

We give an example to solve the driven cavity flow problem. It is solved first at zero Reynolds number (Stokes flow) and then at Reynolds number 100 (Navier Stokes).

The driven cavity problem computes the flow in the unit box $] 0,1[2$, with a zero velocity on left, right and down sides and $(1,0)$ velocity on the upper side.

The velocity and pressure formulation is used first, and then the calculation is repeated with the stream function vorticity formulation.

The Stokes equations in a domain $\Omega=] 0,1\left[^{2}\right.$ are:

$$
\left.\begin{array}{rl}
-\Delta u+\nabla p & =0 \\
\nabla \cdot u & =0
\end{array}\right\} \quad \text { in } \Omega
$$

where $u$ is the velocity vector and $p$ the pressure. For simplicity, let us choose Dirichlet boundary conditions on the velocity, $u=u_{\Gamma}$ on $\Gamma=\partial \Omega$.

A classical way to discretize the Stokes equation with a mixed formulation (see for example Chap. 5 in [10] for details) is to solve the variational problem and then discretize it:

Find $\left(u_{h}, p_{h}\right) \in X_{h}^{2} \times M_{h}$ such that $u_{h}=u_{\Gamma h}$, and such that

$$
\begin{aligned}
\int_{\Omega_{h}} \nabla u_{h} \cdot \nabla v_{h}-\int p_{h} \nabla \cdot v_{h} & =0, \quad \forall v_{h} \in X_{0 h} \\
-\int_{\Omega_{h}} \nabla \cdot u_{h} q_{h} & =0, \quad \forall q_{h} \in M_{h}
\end{aligned}
$$

where $X_{0 h}$ is the space of functions of $X_{h}$ which are zero on $\Gamma$. The velocity space is approximated by $X_{h}$, and the pressure space is approximated by $M_{h}$.

The mesh is constructed by:

\section{mesh $T h=s q u a r e(8,8) ;$}

The labels assigned by square to the bottom, right, up and left edges are respectively 1,2,3, 4 .

We use a classical Taylor-Hood (Sect. 4.3.3 in [10]) element to solve the problem:

The velocity is approximated with the $P_{2}$ elements $\left(X_{h}\right)$, and the the pressure is approximated with the $P_{1}$ elements $\left(M_{h}\right)$ :

$$
X_{h}=\left\{v \in H^{1}(\Omega) / \forall K \in \mathcal{T}_{h} \quad v_{\mid K} \in P_{2}\right\}
$$


and

$$
M_{h}=\left\{v \in H^{1}(\Omega) / \forall K \in \mathcal{T}_{h} \quad v_{\mid K} \in P_{1}\right\}
$$

The finite element spaces and functions are constructed by:

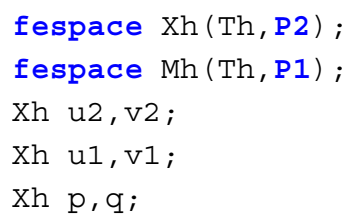

The Stokes operator is implemented as a system-solve for the velocity $(u 1, u 2)$ and the pressure $p$. The test function for the velocity is $(v 1, v 2)$ and $q$ for the pressure, so the variational form $(7)$ in FreeFem ++ language is:

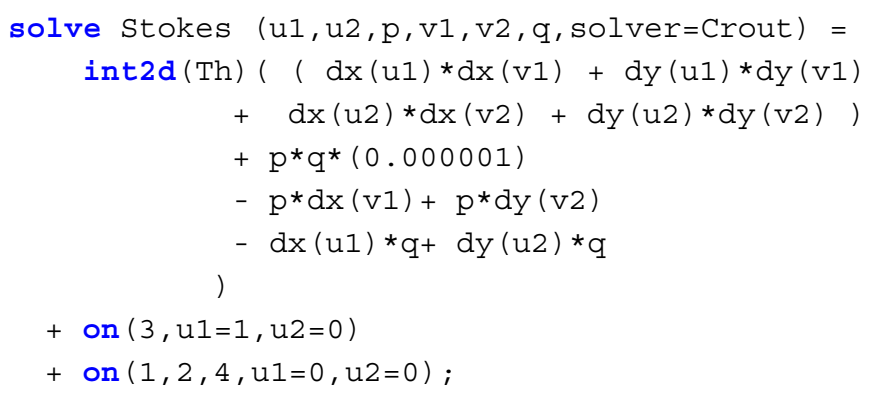

Each unknown has its own boundary conditions. The results of the Stokes problem are shown in Figure 1.

Technical Remark. There is some arbitrary decision here as to where to affect the boundary condition within the linear system (see [9] for more details). Basically the Dirichlet operator (on) should be associated with the unknown which contains it so that the penalization appears on the diagonal of the matrix of the underlying discrete linear system, otherwise it will be ill conditioned.

Remark. Notice that the term $\mathrm{p} * \mathrm{q}^{*}(0.000001)$ is added, because the Crout solver (Chap. 2.6.2 in [7]) needs all the sub-matrices to be invertible.

If the streamlines are required, they can be computed by finding $\psi$ such that $\operatorname{rot} \psi=u$ or better,

$$
-\Delta \psi=\nabla \times u
$$

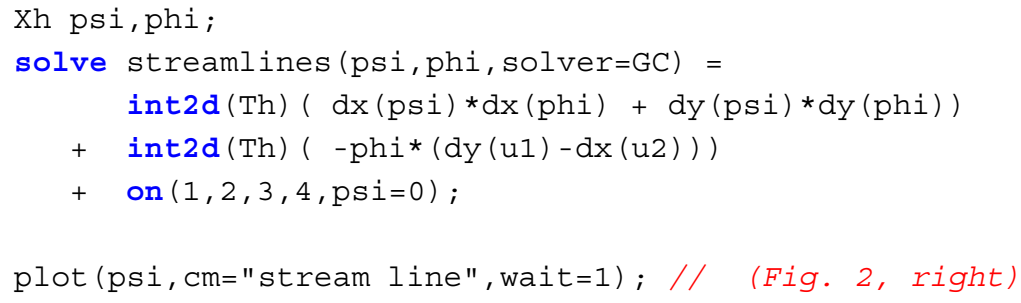

Now the time dependant Navier-Stokes equations are solved

$$
\frac{\partial u}{\partial t}+u \cdot \nabla u-\Delta u+\nabla p=0, \nabla \cdot u=0 .
$$



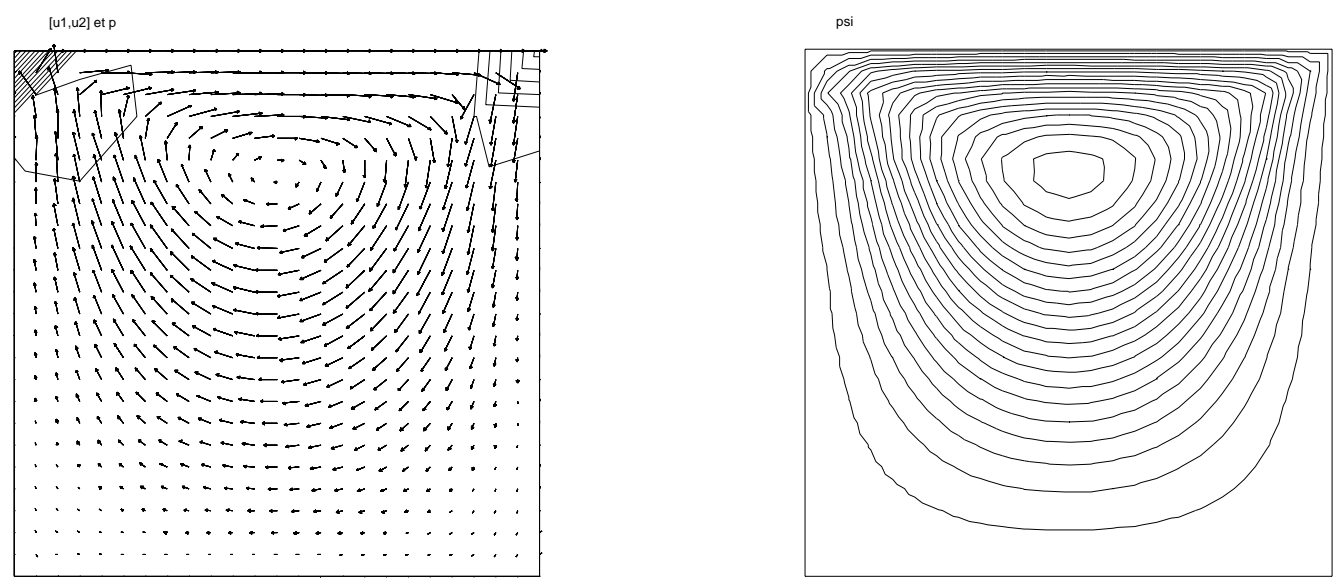

Figure 1. Stokes solution: the left part is the plot of the isovalues of the pressure and the velocity vector field; the right part is the plot of the streamlines.

with the same boundary conditions and with initial conditions $u=0$.

This is implemented by using the convection operator convect for the term $\frac{\partial u}{\partial t}+u \cdot \nabla u$, giving a discretization in time

$$
\begin{aligned}
\frac{1}{\delta t}\left(u^{n+1}-u^{n} \circ X^{n}\right)-\nu \Delta u^{n+1}+\nabla p^{n+1} & =0, \\
\nabla \cdot u^{n+1} & =0 .
\end{aligned}
$$

The term, $u^{n} \circ X^{n}(x) \approx u^{n}\left(x-u^{n}(x) \delta t\right)$ will be computed by the operator "convect", so we obtain

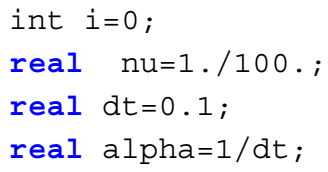

Xh up1, up2;

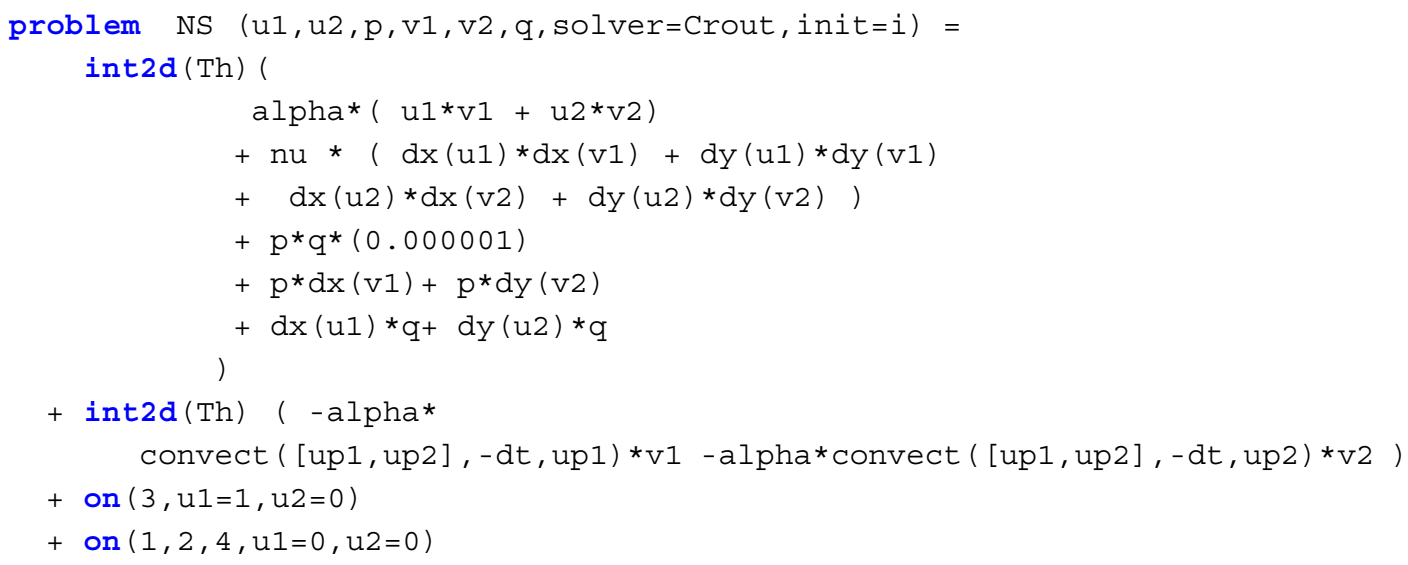



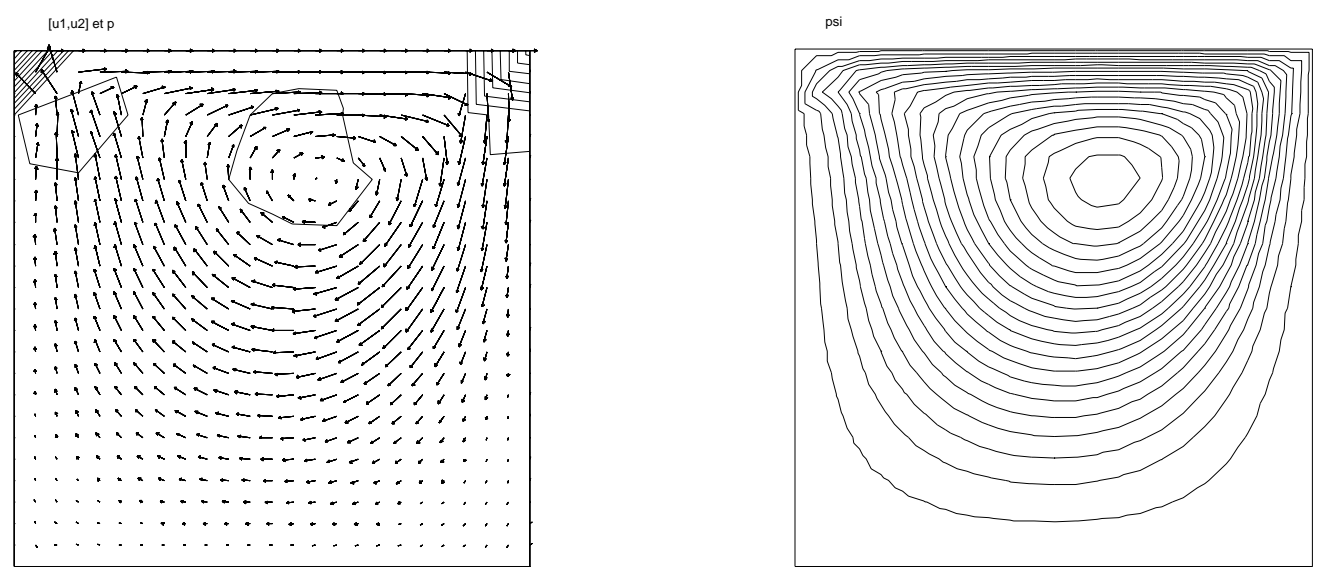

FigurE 2. Navier-Stokes solution at $R e=100$ : the left part is the plot of the isovalue of the pressure and the plot of velocity; the right part is the plot of the streamlines.

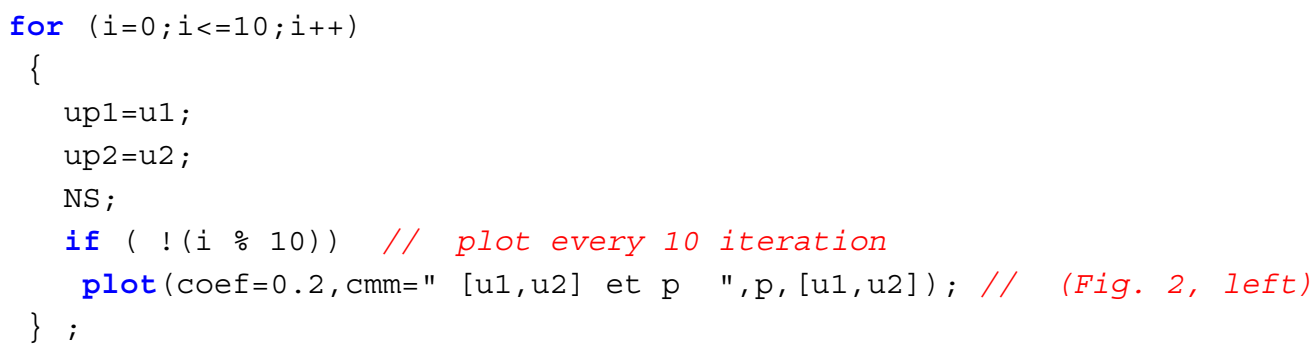

\subsection{Schwarz domain decomposition}

To solve

$$
-\Delta u=f, \text { in } \Omega=\left.\Omega_{1} \cup \Omega_{2} \quad u\right|_{\Gamma}=0
$$

the Schwarz algorithm runs like this:

$$
\begin{aligned}
& -\Delta u_{1}^{m+1}=f \text { in }\left.\Omega_{1} \quad u_{1}^{m+1}\right|_{\Gamma_{1}}=u_{2}^{m} \\
& -\Delta u_{2}^{m+1}=f \text { in }\left.\Omega_{2} \quad u_{2}^{m+1}\right|_{\Gamma_{2}}=u_{1}^{m}
\end{aligned}
$$

where $\Gamma_{i}$ is the boundary of $\Omega_{i}$ and under the condition that $\Omega_{1} \cap \Omega_{2} \neq \emptyset$ and that $u_{i}$ are zero at iteration 1 .

Here we take $\Omega_{1}$ to be a quadrangle, $\Omega_{2}$ a disk and we apply the algorithm starting from zero.

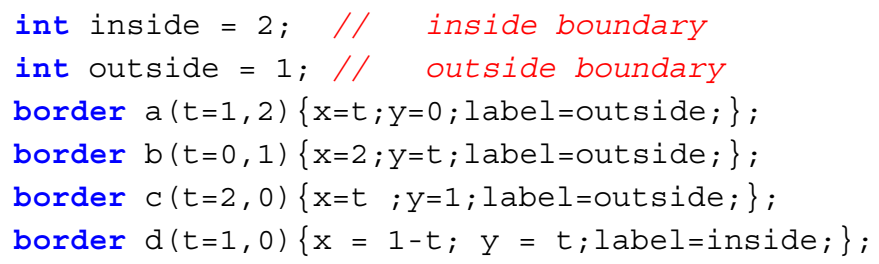




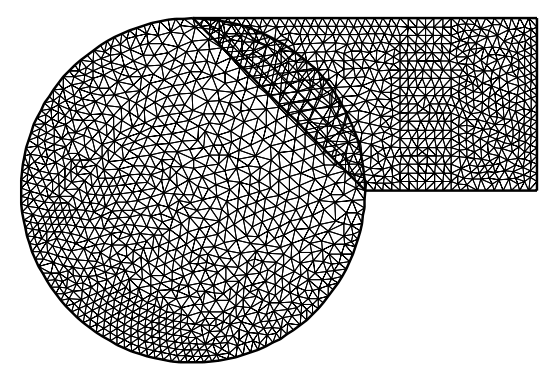

Figure 3. The two overlapping mesh $\mathrm{TH}$ and th.
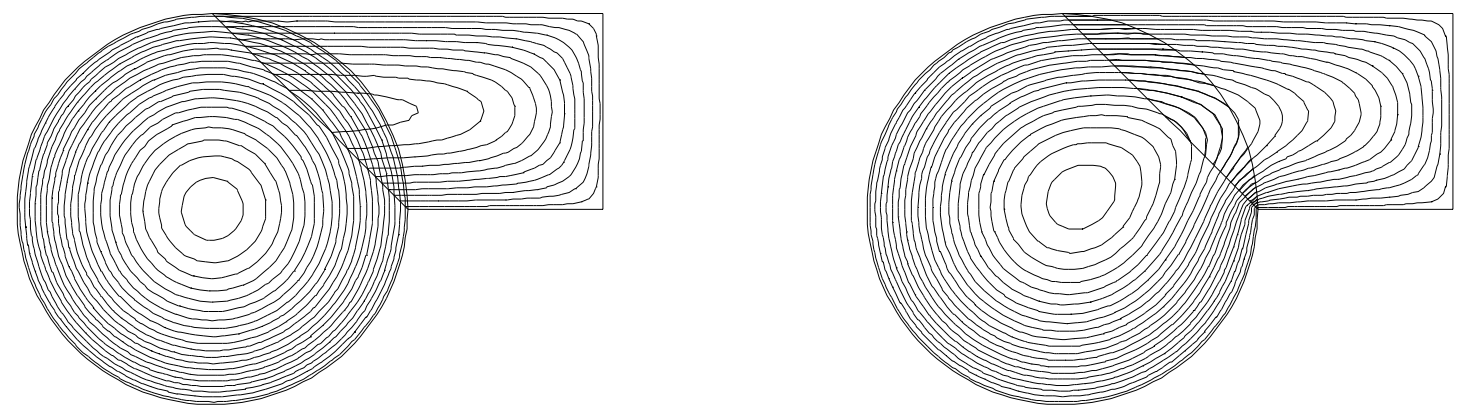

Figure 4. Isovalues of the solution at iteration 0 and iteration 9.

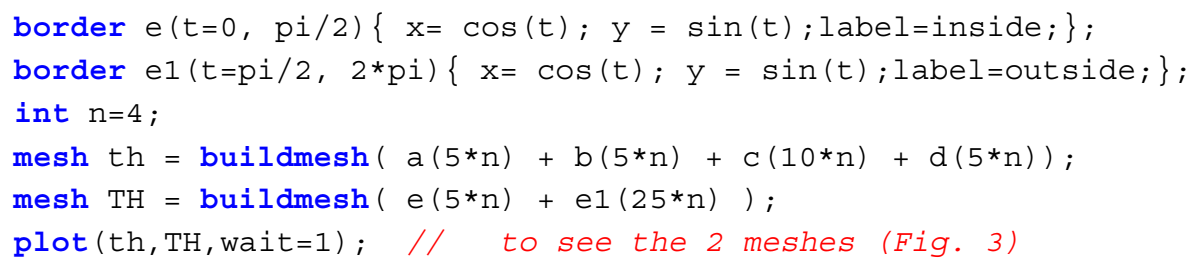

The automatic mesh generator is based on the bamg software [5], and the mathematical background of this mesh generator is avalaible in [4].

The space and problem definition is:

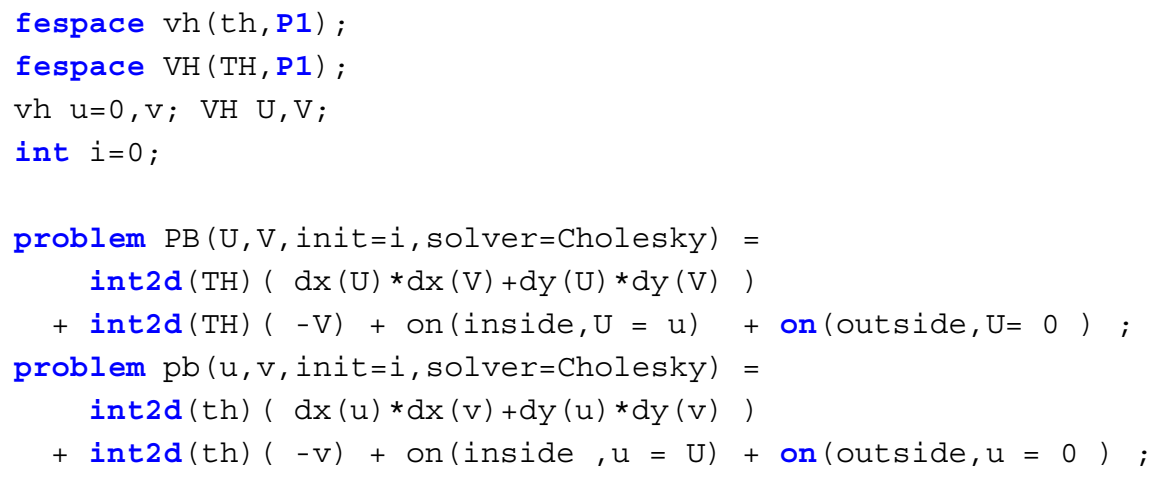


The calculation loop:

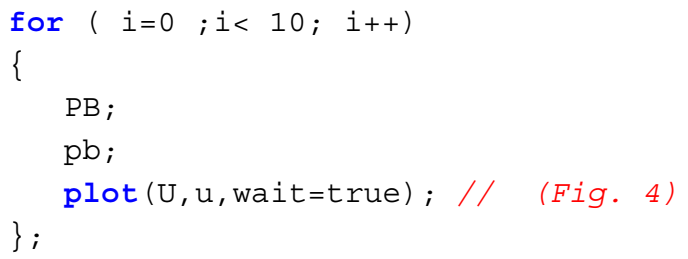

Acknowledgements. Aknowledgements to Olivier Pironneau and Dominique Bernardi for an infinite number of coffee talks on these matters.

\section{REFERENCES}

[1] D. Bernardi, F. Hecht, K. Ohtsuka and O. Pironneau, freefem+documentation. http://www-rocq.inria.fr/Frederic. Hecht/freefem+.htm

[2] P.G. Ciarlet, Basic error estimates for elliptic problems, in Handbook of Numerical Analysis, Vol. II, P.G. Ciarlet and J.-L. Lions Eds., North-Holland (1991) 17-351.

[3] C. Donnelly and R. Stallman, Bison documentation. http://www.gnu.org/bison

[4] P. Frey and P.L. George, Automatic triangulation. Wiley (1996).

[5] F. Hecht, The mesh adapting software: bamg. http://www-rocq.inria.fr/gamma/cdrom/www/bamg/eng.htm INRIA (1998).

[6] F. Hecht and O. Pironneau, freefem++ Manual. http://www-rocq.inria.fr/Frederic.Hecht/freefem++.htm

[7] P. Joly and M. Vidrascu, Quelques méthodes classique de résolution de systèmes linèaires. Collection didactique, INRIA (1994).

[8] J.L. Lions and O. Pironneau, Domain decomposition methods for CAD. C. R. Acad. Sci. Paris Sér. I Math. 328 (1999) 73-80.

[9] B. Lucquin and O. Pironneau, Scientific Computing for Engineers. Wiley (1998).

[10] O. Pironneau, Méthodes des éléments finis pour les fluides. Masson (1988).

[11] N. Wirth, Algorthims + Data Structures = Programs. Prentice Hall (1976).

To access this journal online: www.edpsciences.org 